\title{
AN INTERACTING GALAXY PAIR AT THE ORIGIN OF A LIGHT ECHO
}

\author{
PaOla Merluzzi ${ }^{1}$ And Giovanni Busarello \\ INAF-Osservatorio Astronomico di Capodimonte, Salita Moiariello 16 I-80131 Napoli, Italy
}

Michael A. Dopita And Adam D. Thomas

Research School of Astronomy and Astrophysics, Australian National University, Canberra, ACT 2611, Australia

Chris P. Haines

INAF-Osservatorio Astronomico di Brera, Via Brera 28 I-20121 Milano, Italy

Aniello Grado, Luca Limatola and Amata Mercurio

INAF-Osservatorio Astronomico di Capodimonte, Salita Moiariello 16 I-80131 Napoli, Italy

${ }^{1}$ merluzzi@na.astro.it

\begin{abstract}
In a low-density region of the Shapley supercluster we identified an interacting galaxy pair at redshift $z=0.04865$ in which the Seyfert-2 nucleus of the main galaxy (ShaSS 073) is exciting an extended emission line region (EELR, $\sim 170 \mathrm{kpc}^{2}$ ) in the disk of the less massive companion (ShaSS 622). New integral-field spectroscopy and the multi-band data-set, spanning from far-ultraviolet to far-infrared and radio wavelengths, allowed us to obtain a detailed description of the ShaSS 622-073 system. The gas kinematics shows hints of interaction, although the overall velocity field shows a quite regular rotation in both galaxies, thus suggesting that we are observing their first encounter as confirmed by the estimated distance of $21 \mathrm{kpc}$ between the two galaxy centers. The detected $\sim 2-3 \mathrm{kpc}$ AGN outflow and the geometry of the EELR in ShaSS 622 support the presence of a hollow bi-cone structure. The status and sources of the ionization across the whole system have been analysed through photoionization models and a Bayesian approach which prove a clear connection between the AGN and the EELR. The luminosity of the AGN $\left(2.4 \times 10^{44} \mathrm{erg} \mathrm{s}^{-1}\right)$ is a factor 20 lower than the power needed to excite the gas in the EELR $\left(4.6 \times 10^{45} \mathrm{erg} \mathrm{s}^{-1}\right)$ indicating a dramatic fading of the AGN in the last $3 \times 10^{4} \mathrm{yr}$. ShaSS $073-622$ provides all the ingredients listed in the recipe of a light echo where a high-ionised region maintains memory of a preceding more energetic phase of a now faded AGN. This is the first case of a light echo observed between two galaxies.
\end{abstract}

Keywords: galaxies: Seyfert — galaxies: interactions — galaxies: active — ISM: kinematics and dynamics - ISM: abundances - galaxies:evolution

\section{INTRODUCTION}

A comprehensive and all-purpose description of the evolution of galaxies is one of the most ambitious goals of astrophysicists and a rewarding approach in this investigation consists in the selection and study of samples of galaxies which are experiencing a specific transformation through tidal interaction, ram-pressure stripping and the like - the so called smoking guns. Signatures of an ongoing transformation may be detected in the galaxy colors, star-formation indicators, morphologies and kinematics (e.g Davies et al. 2015a; Smith et al. 2016; Conselice 2014; Rubin et al. 1999), so their detec- tion encompasses the whole spectral range and galaxy regions recommending a multi-band approach together with integral-field spectroscopy (IFS) to effectively understand the physics of the mechanism/s at work either internal or related to the galaxy environment.

One of the features that is more likely associated to an ongoing transformation is the presence of extra-planar ionized gas. This can be driven by ram-pressure stripping (RPS, Gunn \& Gott 1972), galaxy-galaxy interactions (Toomre \& Toomre 1972) or nuclear activity (Stockton et al. 2006). In particular, several active galaxies show regions of ionized gas extending out of 
their disk (e.g. Fu \& Stockton 2009) whose geometry, structure, extent and luminosity provide unique information about the physics of the active galactic nuclei (AGNs) and their interaction with the host galaxy (see King \& Pounds 2015, for a review).

In broad terms, clouds of ionized gas detected in active galaxies either are classified as narrow-line regions (NLRs), having different sizes and morphologies, but generally having a bi-cone structure as predicted by the unified AGN scenario (see Netzer 2015), or as extended emission line regions (EELRs) which are likely produced in merger events, and which are dynamically decoupled from the host galaxy. Such regions may be either photoor shock-ionized.

Although the physics of the AGN has been deeply investigated (see Fabian 2012, for a review), the complexity of this process, which involves the whole properties of the interstellar medium (ISM, dust content and chemical abundances) and stellar populations of the host galaxy, may give rise to very different effects. Extended NLRs (ENLRs) are rather common in luminous quasars (e.g. Hainline et al. 2014), however at lower redshifts few restricted samples of lower luminosity objects presenting ENLRs were recently available to gain new insights into the complex field of AGN variability and structure.

A very specific example of this complexity is the peculiar object nicknamed Hanny's Voorwerp, a cloud of ionized gas located $20 \mathrm{kpc}$ outside the spiral galaxy IC 2497 at redshift $z \sim 0.05$ (Lintott et al. 2009). This has been interpreted as a light echo ionized by an AGN (Rampadarath et al. 2010) which faded dramatically within the last $\sim 10^{5} \mathrm{yr}$. This was the first evidence for such a time scale of AGN variability. To explain the nuclear starburst of IC 2497 (Rampadarath et al. 2010), the huge reservoir of $\mathrm{H}$ I around the galaxy (Józsa et al. 2009), the discrepancy between the level of ionization of the cloud and both the luminosity of the AGN and the nature of the X-ray emission (Lintott et al. 2009; Sartori et al. 2016), Keel et al. (2012b) suggested the following sequence of events. A major merger produced the massive tail of $\mathrm{HI}$ surrounding both the galaxy and Hanny's Voorwerp and triggered the AGN activity. Later, the ionizing luminosity from the AGN activity dropped abruptly leaving Hanny's Voorwerp as the only highly ionized region in the system.

The discovery of this system boosted a systematic search for similar objects. Keel et al. (2012a) identified 19 galaxies with AGN-ionized regions at projected radii $r_{p}>10 \mathrm{kpc}$ out of 18,166 candidates drawn from the SDSS DR 7. Among these, 8 show a strong deficit in ionizing luminosity as well as no evidence of strongly obscured AGN. Almost all of these (probably faded) AGN candidates present signs of interaction or merger and the authors claimed that the observed EELRs are largely photoionized tidal debris. Nevertheless, none of these object has been caught in the act of interacting or merging with a companion. Thus, we are observing the results and not the catalyst.

Schirmer et al. (2013) discovered a class of 29 Seyfert-2 galaxies in the redshift range $z=0.2-0.6$ from the SDSS DR 8. These are characterized by bright NLRs, possibly surrounded by ENLRs. Due to their high-luminosity [O III] $\lambda 5007$ emission, these objects have received the nickname green beans (GBs) similarly to the emissionline compact galaxies found by Cardamone et al. (2009) which have been dubbed green peas. Although having [O III] luminosities two orders of magnitude higher with respect to the Keel et al.'s sample and spanning a different redshift range, they share the property of that sample in that the AGN luminosity is insufficient to power the ENLRs, and in particular the [O III] flux. This suggests that these objects as well may be classified as AGN ionization echoes. Schirmer et al. (2013) have also found hints of interaction with neighbouring galaxies in their GBs sample.

Objects such as Hanny's Voorwerp or the GBs are rare. This can be either due to the intrinsic low probability of such phenomena or to their short duration. However, with respect to IC 2497, Keel et al. (2012b) pointed out that it is "unlikely that a very rare event would be represented so close to us" which rather suggests that this is a common phenomenon in AGNs. Therefore, detailed study of these few cases is required in order to improve our understanding both of AGN physics and its impact on galaxy evolution.

In this work, we study a galaxy pair in a very early phase of interaction. The more massive galaxy has a Seyfert-2 nucleus which is illuminating a vast area of the disc of the companion galaxy. Through a detailed analysis of the properties of this system we aim at shedding light on the origin and nature of the EELRs. This galaxy pair, at $z \sim 0.05$, belongs to a relatively sparse region in the Shapley Supercluster. Our analysis is based on new IFS observations and complementary multi-band data from the Shapley Supercluster Survey (ShaSS, Merluzzi et al. 2015), as well as from literature.

We describe the selection and properties of the target in Sect. 2 and the IFS observations in Sect. 3, then in Sect. 4 the modelling of the stellar component of the spectrum is briefly explained. Gas kinematics and physical properties are derived and analyzed in Sects. 5 and 6 , respectively. Photoionization modelling is detailed in Sect. 7, while the structure of the system is described in Sec. 8. We discuss our results in Sect. 9 and summarize them in Sect. 10.

Throughout the paper we adopt a cosmology with $\Omega_{M}=0.3, \Omega_{\Lambda}=0.7$, and $\mathrm{H}_{0}=70 \mathrm{~km} \mathrm{~s}^{-1} \mathrm{Mpc}^{-1}$. According to this cosmology 1 arcsesc corresponds to $0.96 \mathrm{kpc}$ 


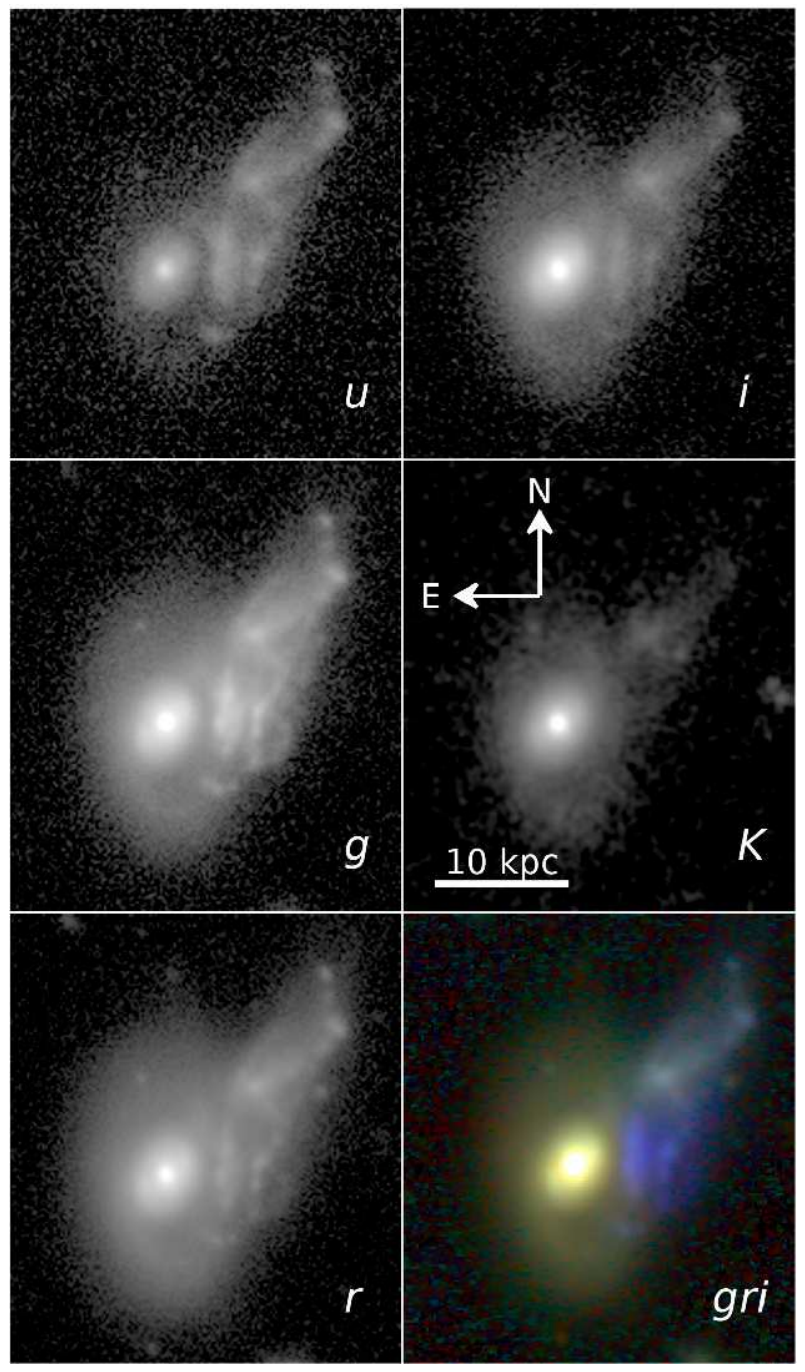

Figure 1. From top to bottom and from left to right: VST ugri bands and VISTA $K$-band images of the ShaSS 073-622 system. ShaSS 073 is the brightest galaxy near the center of the frame and ShaSS 622 is the gas-rich galaxy NW to it. Bottom right: color composite image with VST gri bands assigned to BGR channels. The bright blue area indicates high $g$-band flux due to strong [O III] emission. North is up and East left in all panels. The orientation of this figure is adopted for all the other figures in this work unless differently specified.

at the target redshift.

\section{THE SHASS 073-622 SYSTEM}

\subsection{Target selection}

Our target has been identified as part of the Shapley Supercluster Survey (ShaSS, Merluzzi et al. 2015). This survey covers $23 \mathrm{deg}^{2}$ centered on the Shapley Supercluster core $(z \sim 0.05)$ and combines observations in the ESO-VST ugri filters ${ }^{1}$ with ESO-VISTA $K$-band imag- ing. The ShaSS imaging has a resolution $\lesssim 1 \mathrm{kpc}^{2}$ at the supercluster redshift which allows us to study the structure of the individual supercluster galaxies.

We exploited the image quality to search for galaxies undergoing transformation and to identify candidates for further IFS observations. All the targets selected for the IFS were drawn from the spectroscopic catalogue of ShaSS which is 80 per cent complete down to $i=17.6\left(\mathrm{~m}^{\star}+3\right)$. All these galaxies are supercluster members, are fully resolved in the optical images and display disturbed morphologies, such as asymmetry and tails, hints of extraplanar emission as well as evidence of star-forming knots. About 80 galaxies satisfy at least two of these criteria. After this visual selection, the galaxies are targeted with a 45-minute exposure with WiFeS which allows to ascertain which of them actually present extraplanar emission and then become the high-priority targets in our investigation. At present 17 supercluster galaxies have been observed. They belong to different environments, from dense cluster cores to the regions where cluster-cluster interactions are taking place, and out to the much less populated areas.

The system ShaSS $073-622^{3}$ at $z=0.04865$ was chosen from the above sample because it appeared to be an interacting pair with one member (ShaSS 073) hosting a Seyfert-2 nucleus (Véron-Cetty \& Véron 2001). This was considered a promising target to study the early phase of the formation of EELRs.

The ugriK ShaSS imaging of our object is shown in Fig. 1 . In the $g$ - and $r$-band images the main galaxy, ShaSS 073, appears as a barred galaxy with an external (slightly asymmetric) ring (e.g. Buta 2013, see Sect. 2.2.1). Note that in all filters the central galaxy presents a star-like nucleus showing that the galaxy hosts an AGN.

A color composite image of the ShaSS 073-622 system with the VST gri bands assigned to BGR channels is also shown in Fig. 1 (bottom right panel) which emphasizes the most striking feature of this system - the bright blue area West of the ShaSS 073 nucleus, due to strong [O III] emission (contributing to the $g$ band at this redshift).

The orientation of Fig. 1 is adopted for all the other figures in this work unless differently specified.

\footnotetext{
2 The FWHM of the optical images correspond to $\sim 0.8, \sim 0.7$, $\sim 0.6, \sim 0.9$ and $\sim 0.9$ arcsec. for the ugriK wavebands.

3 The system consists of the two galaxies ShaSS 423045073 and ShaSS 423045622 drawn from the Shapley Supercluster Survey (Merluzzi et al. 2015; Mercurio et al. 2015). In the paper we use an abbreviated form of these identifications.
}

\footnotetext{
1 The VST images have been processed at the VST Data Center using the VST-Tube pipeline (Grado et al. 2012).
} 
Table 1. The galaxies

\begin{tabular}{lll}
\hline \multicolumn{1}{c}{ Property } & ShaSS 423045073 & ShaSS 423045622 \\
& & \\
\hline Coordinates $^{1,2}$ & $131632.58-311218.5$ & $131632.02-311211.5$ \\
(J2000) & & \\
& & \\
Magnitudes $^{3,4,5}$ & & $17.68 \pm 0.04$ \\
$u^{(\mathrm{a})}$ & $17.70 \pm 0.04$ & $16.64 \pm 0.02$ \\
$g^{(\mathrm{a})}$ & $16.20 \pm 0.02$ & $16.87 \pm 0.02$ \\
$r^{(\mathrm{a})}$ & $15.47 \pm 0.02$ & $17.03 \pm 0.02$ \\
$i^{(\mathrm{a})}$ & $15.11 \pm 0.02$ & $14.95 \pm 0.03$ \\
$K^{(\mathrm{a})}$ & $12.22 \pm 0.03$ & \\
$\mathrm{~W}^{(\mathrm{b})}$ & $11.69 \pm 0.02^{(\mathrm{c})}$ & \\
$\mathrm{W}^{(\mathrm{b})}$ & $10.59 \pm 0.02^{(\mathrm{c})}$ & \\
$\mathrm{W}^{(\mathrm{b})}$ & $7.19 \pm 0.02^{(\mathrm{c})}$ & \\
$\mathrm{W}^{(\mathrm{b})}$ & $4.79 \pm 0.03^{(\mathrm{c})}$ & \\
$\mathrm{F}_{60 \mu \mathrm{m}}$ & $0.40 \pm 0.05 \mathrm{Jy}^{(\mathrm{c})}$ & \\
$\mathrm{F}_{1.4 \mathrm{GHz}}$ & $3.9 \pm 0.6 \mathrm{mJy}^{(\mathrm{c})}$ & \\
\end{tabular}

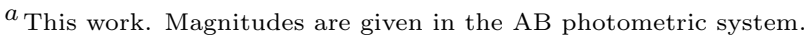

${ }^{b}$ Magnitudes are given in the Vega photometric system.

${ }^{c}$ Centered on ShaSS 073 .

Note-Sources: ${ }^{1}$ Merluzzi et al. (2015); ${ }^{2}$ Mercurio et al. (2015); ${ }^{3}$ Wright et al. (2010); ${ }^{4}$ Neugebauer et al. (1984), ${ }^{5}$ Condon et al. (1998)
}

\subsection{Properties of the ShaSS 073-622 system}

In Fig. 2, we show the ShaSS stellar mass surface density map derived from the flux at $3.4 \mu \mathrm{m}$ from the WISE data (see Merluzzi et al. 2015). We have indicated the position of our target as a white star together with four other supercluster galaxies with confirmed ongoing RPS (see Merluzzi et al. 2013, 2016). While the four galaxies affected by RPS are associated with clusters, ShaSS 073 is located in a low density region. The photometric properties of the galaxies ShaSS 073 and ShaSS 622 are listed in Table 1 . The optical and $K$-band magnitudes are derived from the ShaSS imaging as explained in Sect. 2.2.1. The projected distance between the two galaxy centers is $\sim 10$ arcsec and the difference of their radial velocity is $\sim 130 \mathrm{~km} \mathrm{~s}^{-1}$.

The source is detected in all four channels of the Wide-field Infrared Survey Explorer (WISE, Wright et al. 2010). The WISE emission in all four bands (W1-W4) is centered on the nucleus of ShaSS 073, suggesting that all of the emission in these bands is due to the primary galaxy rather than the companion. The galaxy has $\mathrm{W} 1-\mathrm{W} 2=1.1, \mathrm{~W} 2-\mathrm{W} 3=3.4$, $\mathrm{W} 3-\mathrm{W} 4=2.4$, while normal quiescent or star-forming galaxies have $\mathrm{W} 1-\mathrm{W} 2 \sim 0.0$. Stern et al. (2012) use $\mathrm{W} 1-\mathrm{W} 2>0.80$ as a criterion to select AGNs. Jar-

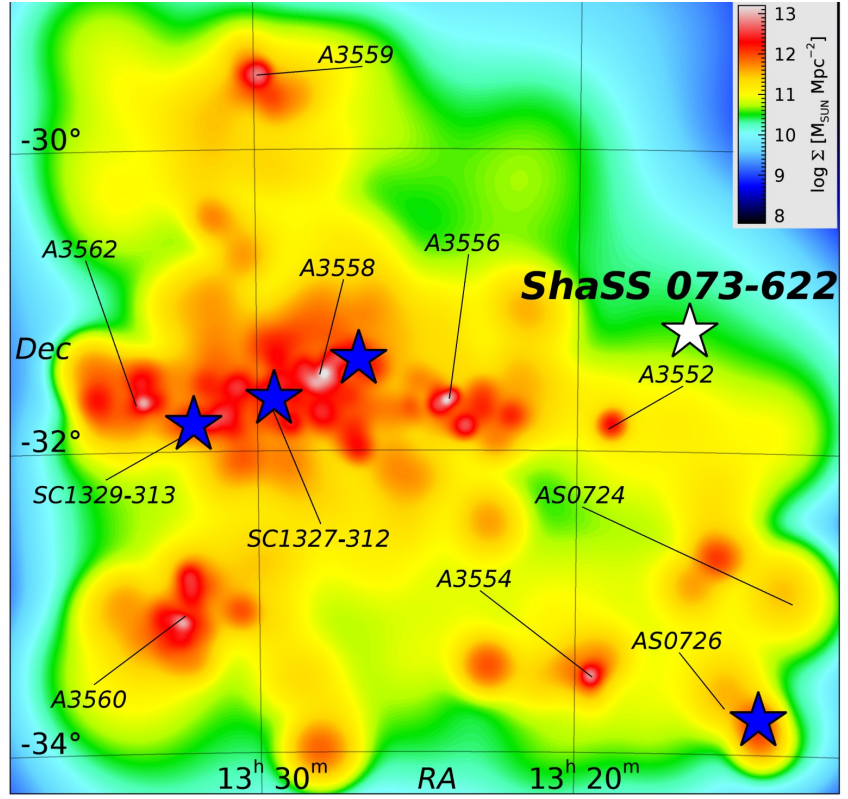

Figure 2. The ShaSS stellar mass surface density map in $\mathrm{M}_{\odot} \mathrm{Mpc}^{-2}$ derived from the flux at $3.4 \mu \mathrm{m}$ (Merluzzi et al. 2015). Abell clusters and groups are labelled by black straight lines pointing on the X-ray center for all systems except AS 0726. The upper right-hand corner is not covered by ShaSS. Blue stars identify the location of four galaxies in the clusters with ascertained ongoing RPS. The white star indicates the location of ShaSS 073-622 system in a low-density environment.

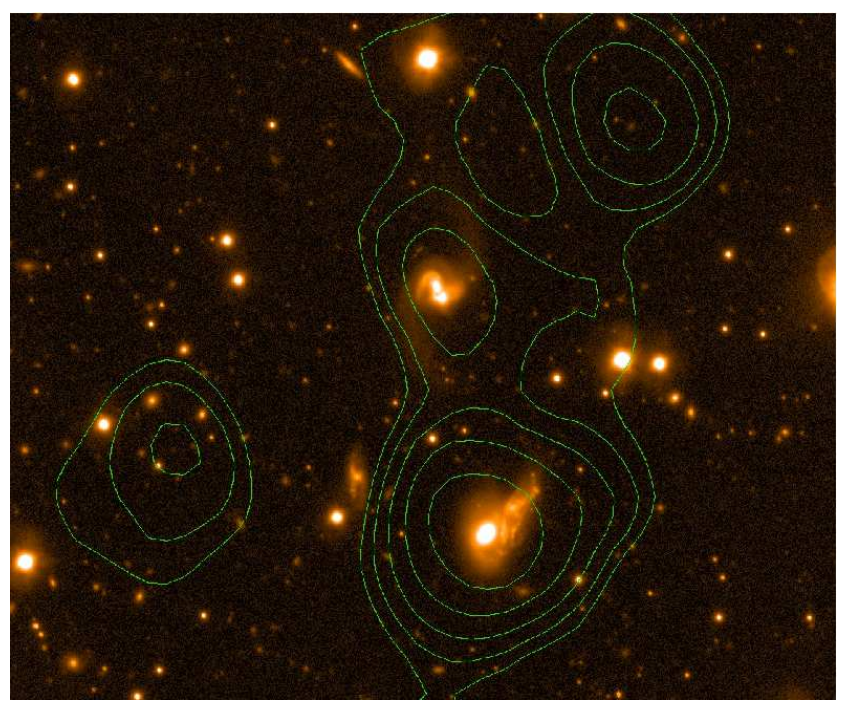

Figure 3. NVSS radio contours overlaid on the VST $r$-band image. The ShaSS 073-622 system is in the lower part of the image, while the galaxy near the center is at $z \sim 0.07$ and is not interacting with it.

rett et al. (2011) select AGNs as lying within the box: $2.2<(\mathrm{W} 2-\mathrm{W} 3)<4.2,0.1 \times(\mathrm{W} 2-\mathrm{W} 3)+0.38<$ $(\mathrm{W} 1-\mathrm{W} 2)<1.7$ where is also located our system.

The system has been detected by IRAS which measures a flux of $\mathrm{F}_{60 \mu m}=0.40 \pm 0.05 \mathrm{Jy}$. It corresponds to 
source IRAS F13138-3056 in the IRAS Faint Source Catalog (Moshir et al. 1990), which is centered $\sim 28 \operatorname{arcsec}$ $\mathrm{E}$ of ShaSS 073, but within the $1 \sigma$ uncertainty in position ( $\sim 20 \operatorname{arcsec}$ in RA and $\sim 7 \operatorname{arcsec}$ in Dec). GALEX NUV and FUV channels also reveal the system, tracing the gas of ShaSS 622 .

Our source has been also detected by the NRAO VLA Sky Survey (NVSS) which covers at $1.4 \mathrm{GHz}$ the entire sky north of $-40 \mathrm{deg}$ declination. In Fig. 3 the NVSS radio contours are overlaid on the VST $r$-band image. These show that the radio emission is centered on the main galaxy. The measured flux, $\mathrm{F}_{1.4 \mathrm{GHz}}=$ $3.9 \pm 0.6 \mathrm{mJy}$, corresponds to a luminosity of $\mathrm{L}_{1.4 \mathrm{GHz}}=$ $(2.20 \pm 0.34) \times 10^{22} \mathrm{~W} \mathrm{~Hz}^{-1}$. This is insufficient for it to be classed as a radio-loud AGN (Yun et al. 2001).

Taking into account both the IR and radio data, we note that the ratios $\mathrm{F}_{22 \mu \mathrm{m}} / \mathrm{F}_{1.4 \mathrm{GHz}}=25.9$ and $\mathrm{F}_{60 \mu \mathrm{m}} / \mathrm{F}_{22 \mu \mathrm{m}}=3.986$ are indicative of an AGN (Dale \& Helou 2002; Haines et al. 2011; Dale et al. 2014). The galaxy however, lies on the $\mathrm{F}_{70 \mu \mathrm{m}} / \mathrm{F}_{1.4 \mathrm{GHz}}$ (see below) FIR-radio relation for normal star-forming galaxies (Haines et al. 2011), rather than radio-loud galaxies. This suggests that the radio emission is mostly due to star formation. Assuming the relation $\mathrm{SFR}=\mathrm{L}_{1.4 \mathrm{GHz}} /\left(3.44 \times 10^{21} \mathrm{~W} \mathrm{~Hz}^{-1}\right)$, then the measured flux would imply a star formation rate $\mathrm{SFR}=6.4 \pm$ $1.0 \mathrm{M}_{\odot} \mathrm{yr}^{-1}$.

We fit the far-infrared emission by the $\log \left(\mathrm{L}_{\mathrm{TIR}}\right)=10.75$ model of Rieke et al. (2009). Constraining this model with the $\mathrm{F}_{60 \mu \mathrm{m}}$ value, we predict a $70 \mu \mathrm{m}$ flux of $\mathrm{F}_{70 \mu \mathrm{m}}=0.491 \mathrm{Jy}$ for the whole system, and $2.4 \mathrm{mJy}$ at $1.4 \mathrm{GHz}$. This model would also predict a $24 \mu \mathrm{m}$ flux of just $33.4 \mathrm{mJy}$, which is only $28 \%$ of the 117.5 mJy flux level actually observed. This shortfall suggests that much of the $24 \mu \mathrm{m}$ (or W4) emission is due to the AGN component rather than star formation, even if all the $60 \mu \mathrm{m}$ emission is assumed to come from star formation.

\subsubsection{Estimates of ugriK magnitudes and stellar masses}

Estimating the optical and $K$-band magnitudes of the two galaxies is not straightforward due to their spatial overlap which affects a large fraction $(\sim 40 \%)$ of their disks. To separate the flux of ShaSS 073 from the companion, we take advantage of the sharp division between the two gaseous discs (see Sects. 5, 6, 7 and Figs. 8, 13).

To make the measurements of fluxes easier, we isolated a frame of 40 arcsec side around the two galaxies, in such a way that there are very few and faint external sources, which are easily masked out (although contributing very little to the total counts). A line through the center of ShaSS 073 and oriented at P.A. $\sim 13^{\circ}$ was adopted to separate the western half of ShaSS 073 which overlaps with the companion from the virtually unper- turbed eastern half. We then derived the magnitude of ShaSS 073 integrating the counts after masking out the western side of the frame with respect to the above line. The total flux from the galaxy was set to twice that flux. Other, more 'refined' masking shapes lead to changes at the level of $0.01 \mathrm{mag}$ which are certainly small considering our ignorance of the 'hidden' part of the galaxy. For ShaSS 622, we integrated the counts of the whole frame and subtracted the total counts estimated for ShaSS 073. With this method we estimated the magnitudes in the $u, g, r, i$ and $K$ bands given in Table 1 .

The resulting colors for ShaSS 073 are $u-g=1.50$, $g-r=0.73$ and $r-i=0.36$, which are consistent with a S0-Sa galaxy (see Fukugita et al. 1995). Taking into account the presence of the bar and the external ring, we classify ShaSS 073 as (R)SB0a (e.g. Buta 2013). The colors of ShaSS $622(u-g=1.04, g-r=-0.23$ and $r-i=-0.16)$ are clearly determined by the illumination from the AGN. If we consider only the areas outside of the ionization cone (see Sect. 6) the colors become $u-g=1.35, g-r=0.63$ and $r-i=0.27$, which are typical of intermediate/late spirals (Sb/Sbc) (Fukugita et al. 1995).

From the above $g$ and $i$ magnitudes and with the adopted cosmology, we estimate for ShaSS 073 a stellar mass of $\mathrm{M}^{*}=5.7 \times 10^{10} \mathrm{M}_{\odot}$ adopting the calibration of GAMA (Taylor et al. 2011). In doing this, we neglect the AGN contribution to the $g$ and $i$ fluxes, as suggested by the spectral energy distribution (see Fig. 4).

The magnitudes and colors of ShaSS 622 are affected by the illumination from the AGN and do not reflect the properties of the stellar populations, so that we cannot use them to estimate the stellar mass. Instead, for the morphological types of our galaxies and their typical colors, we can assume that their $K$-band mass-to-light ratios are similar, according to Bell \& de Jong (2001, see their Fig. 3), therefore we adopted the ratios of $K$ band fluxes as a proxy of their mass ratio. We first removed the AGN contribution from the $K$-band flux of ShaSS 073 as follows. From the spectral energy distribution presented in Fig. 4, the AGN contributes $~ 30 \%$ of the flux at $\sim 2 \mu \mathrm{m}$. To account for this, we multiplied by 0.7 the flux in a circle of diameter twice the FWHM ( 0.9 arcsec) of the $K$-band image, obtaining a total $K$ band magnitude of $12.41 \mathrm{mag}$. From the difference of the $K$-band magnitudes we then derived a stellar mass ratio of $\mathrm{M}^{*}{ }_{073} / \mathrm{M}^{*}{ }_{622} \sim 10$. This classifies the potential merger between our two galaxies as a minor or intermediate merger (Hopkins et al. 2009; Wang et al. 2012).

\subsection{Spectral energy distribution of ShaSS 073}




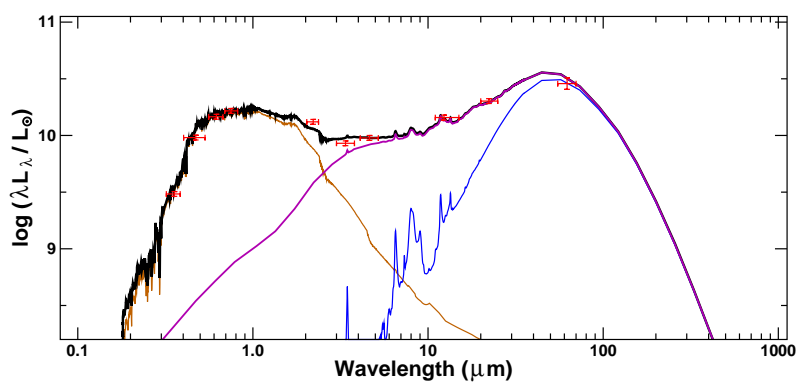

Figure 4. The integrated spectral energy distribution of ShaSS 073 (red points with error bars) combining VST optical, VISTA $K$-band, WISE W1-W4 bands and IRAS $60 \mu \mathrm{m}$ flux measurements. The black curve shows the best-fit model SED, consisting of a template elliptical galaxy SED (brown curve) from Polletta et al. (2007), combined with the twoparameter infrared SED from Dale et al. (2014), which includes emission from both an AGN and star-formation (magenta curve). The blue curve shows the contribution from just the star formation component in this mixed model.

Fig. 4 shows the spectral energy distribution of ShaSS 073 with measured luminosities in ten optical and infrared bands (red points): the ugri photometry from VST, the VISTA $K$-band photometry, the WISE W1W4 bands and the IRAS $60 \mu \mathrm{m}$ band. The GALEX photometry is not used as the NUV and FUV emission spatially coincides with the companion galaxy and the ionized gas cloud rather than ShaSS 073.

The mid-infrared continuum can be well described as a featureless power-law over $3-60 \mu \mathrm{m}$, consistent with dust heating powered by nuclear activity (Stern et al. 2005; Donley et al. 2012).

The brown curve shows the best-fit template elliptical galaxy SED from Polletta et al. (2007), which reproduces well the observed optical photometry as well as the absence of any significant ultraviolet emission from ShaSS 073 in the GALEX images. This leaves little scope for unobscured emission from star formation or an AGN. We instead fit the infrared photometry using the two-parameter semi-empirical model SEDs of Dale et al. (2014), which include contributions from both an AGN and normal star formation. The infrared emission from star formation is modelled using slightly modified versions of the Dale \& Helou (2002) templates, which represent emission from dust exposed to a wide range of heating intensities $0.3 \leq \mathcal{U} \leq 10^{5}$ (where $\mathcal{U}=1$ corresponds to the interstellar radiation field in the solar neighbourhood). The global dust emission of the galaxy is then modelled as a power-law distribution of dust mass $\left(M_{d}\right)$ heated by different radiation intensities, $d M_{d} \propto \mathcal{U}^{-\alpha_{S F}} d \mathcal{U}$, giving a series of templates parametrized by an exponent $\alpha_{S F}$ in the range $0-4$. A second parameter is introduced to account for the infrared emission from an unobscured AGN component. For this purpose, the median mid-infrared spectrum of Shi et al. (2013) is used, which combines Spitzer Infrared
Spectrograph (IRS) observations and MIPS photometry $(24,70$ and $160 \mu \mathrm{m})$ of Palomar-Green quasars, and carefully removes any far-infrared contribution from star formation. Dale et al. (2014) produce a suite of infrared SEDs in which the emission comes from a linear combination of AGN and star-forming templates, in which the AGN contributes between 0 and $100 \%$ of the emission over the 5-20 $\mu \mathrm{m}$ wavelength range, spaced at $5 \%$ intervals.

We fitted these mixed AGN+SF model SEDs to the observed infrared emission from the $K$-band through to the IRAS $60 \mu \mathrm{m}$ band, after subtracting the contribution from the best-fit elliptical template in each pass-band. The best-fit overall SED combining the elliptical SED and the mixed AGN+SF model is shown by the black curve in Fig. 4. We were able to reproduce well the overall power-law form of the infrared SED over 3-60 $\mu \mathrm{m}$ as a model (magenta curve) in which $85 \%$ of the $5-20 \mu \mathrm{m}$ emission comes from the AGN, and the remaining $15 \%$ comes from a star-formation template with $\alpha_{S F}=1.19$ (blue curve), indicating a significant contribution from warm dust heated to $50-100 \mathrm{~K}$. The fraction from starformation cannot be much lower than $15 \%$ as such models cannot reproduce the $60 \mu \mathrm{m}$ flux, while reducing the contribution from the AGN results in models that predict too little emission in the W2 band and too much at $60 \mu \mathrm{m}$.

The WISE $4.6 \mu \mathrm{m}$ (W2) band provides the key constraint on the bolometric AGN luminosity. The photospheric emission from evolved stars in the template elliptical SED (brown curve) drops rapidly in the nearinfrared above $2 \mu \mathrm{m}$, and while the PAH complexes at 6-8 $\mu \mathrm{m}$ and $12 \mu \mathrm{m}$ from H II regions can dominate the mid-infrared emission in normal star-forming galaxies, the emission linked to obscured star-formation drops off rapidly below $5 \mu \mathrm{m}$ (blue curve) and is negligible in the W2 band. It is thus not possible to fit the level of emission observed in the $4.6 \mu \mathrm{m}$ W2 band through any combination of normal galaxy template SED and dust obscured star-formation. Instead, the flat power-law emission from a torus of hot dust, which absorbs the UVoptical radiation coming from the accretion disk and reemits in the infrared, is required to produce the observed SED over 2-30 $\mu \mathrm{m}$, dominating the emission in all four WISE bands. For the W2 band, $88 \%$ of the modelled emission (black curve) is predicted to come from the AGN component (magenta curve) and just $12 \%$ from the photospheric emission from evolved stars (brown curve).

The total infrared luminosity integrated over $3-1100 \mu \mathrm{m}$ from the best-fit $\mathrm{AGN}+\mathrm{SF}$ model is $8.0 \times 10^{10} L_{\odot}$. The far-infrared emission from the AGN component drops rapidly beyond $30 \mu \mathrm{m}$, and so essentially all the IRAS $60 \mu \mathrm{m}$ flux is predicted to come from obscured star-formation, thus providing a constraint on 
the obscured SFR across the galaxy.

Based on the best-fit AGN SED model from Dale et al. (2014), the contributions from the AGN at the $5 \mu \mathrm{m}$ luminosity $\left(\lambda \times \mathrm{L}_{\lambda}\right)$ accounts for the $90 \%$ of the total galaxy emission and is estimated as $3.09 \times 10^{43} \mathrm{erg} \mathrm{s}^{-1}$. Lacy et al. (2015) provide a relation between the $5 \mu \mathrm{m}$ luminosity and the bolometric luminosity of AGNs, based on a bolometric correction of a factor 8.0 (assuming Type I AGN from Richards et al. 2006). This gives us an estimate of the AGN bolometric luminosity as being $2.47 \times 10^{44} \mathrm{erg} \mathrm{s}^{-1}$.

The $6 \mu \mathrm{m}$ luminosity of the AGN component has been shown to correlate linearly with the X-ray luminosity of AGNs (see Mateos et al. 2015). This lends support to the use of the $6 \mu \mathrm{m}$ luminosity as a proxy for AGN bolometric luminosity. At $6 \mu \mathrm{m} 93 \%$ of the total luminosity of ShaSS 073 should be coming from the AGN component corresponding to a luminosity of $3.72 \times 10^{43} \mathrm{erg} \mathrm{s}^{-1}$. The bolometric correction at $6 \mu \mathrm{m}$ should be a factor 6.5 (Richards et al. 2006), giving us an estimate of the bolometric luminosity of $2.42 \times 10^{44} \mathrm{erg} \mathrm{s}^{-1}$, in remarkable agreement with the previous value.

\section{INTEGRAL-FIELD SPECTROSCOPY: OBSERVATIONS AND DATA PROCESSING}

The spectroscopic data on ShaSS 073-622 system were obtained during the night of 23 May 2015 using the Wide-Field Spectrograph (WiFeS, Dopita et al. 2007, 2010). This instrument is mounted at the Nasmyth focus of the Australian National University 2.3m telescope located at the Siding Spring Observatory, Australia. WiFeS is an image-slicing integral-field spectrograph that records optical spectra over a contiguous $25^{\prime \prime} \times 38^{\prime \prime}$ field-of-view. The spectra were acquired in 'binned mode', providing $25 \times 38$ spaxels each of $1^{\prime \prime} \times 1^{\prime \prime}$ size. WiFeS is a double-beam spectrograph providing independent channels for each of the blue and the red wavelength ranges. We used the B3000 and R3000 gratings, allowing simultaneous observations of the spectral range from $\sim 3300 \AA$ to $\sim 9300 \AA$ with an average resolution of $\mathrm{R}=2900$. For further details on the WiFeS instrument see Dopita et al. (2007, 2010).

Two exposures of $45 \mathrm{~min}$ each were obtained on-target (T). We also acquired the spectrum of a nearby sky reference region $(\mathrm{S})$ with three $22.5 \mathrm{~min}$ exposures in order to permit accurate sky subtraction. We adopted the S-T-S-T-S strategy for the observation sequence. Absolute photometric calibration of the data cubes was made using the STIS spectrophotometric standard stars HD 074000 and HD $111980^{4}$. In addition the B-type

\footnotetext{
${ }^{4}$ Available at : www.mso.anu.edu.au/ bessell/FTP/Bohlin2013/GO12813.html
}

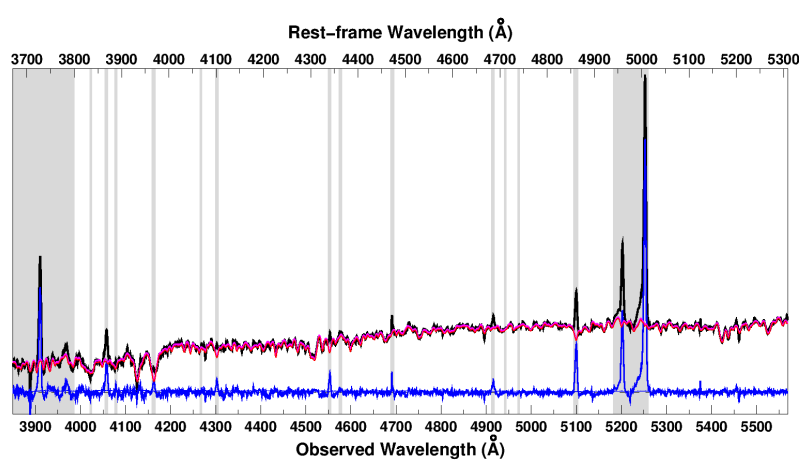

Figure 5. The stellar component fit to data from the blue arm of WiFeS. The thick black curve shows the input spectrum, coming from a $6 \times 7$ spaxels region around the photometric center of the galaxy. The magenta curve shows the resultant best-fit stellar spectrum (see text). The shaded regions indicate the wavelength ranges excluded from the fitting process, including the masks for emission lines. The residual emission component (thick blue curve) reveals clear emission at $[\mathrm{O} \mathrm{II}] \lambda 3729, \mathrm{H} \zeta, \mathrm{H} \epsilon, \mathrm{H} \delta, \mathrm{H} \gamma, \mathrm{H} \beta$ and [O III] $\lambda \lambda 4959-5007$ in the blue arm.

telluric standard HIP 62448 was observed twice during the night. Separate corrections for $\mathrm{OH}$ and $\mathrm{H}_{2} \mathrm{O}$ telluric absorption features were made. During the observations of the target, the seeing averaged $0.8-1.3$ arcsec, which is well matched to the 1.0 arcsec pixels of the spectrograph. Arc and bias frames were also taken between each science exposure. Internal continuum lamp flat fields and twilight sky flats were taken to provide sensitivity corrections for both the spectral and spatial directions.

The data were reduced using the PyWiFes pipeline (Childress et al. 2014). In brief, this produces a data cube which has been wavelength calibrated, sensitivity corrected (including telluric corrections), photometrically calibrated, and from which the cosmic ray events have been removed. Because the PyWiFes pipeline uses a full optical model of the spectrograph to provide the wavelength calibration, the wavelength solution is good across the whole field, and does not rely on any interpolation of the data, since each pixel is assigned a precise wavelength and spatial coordinate. The only interpolation occurs when constructing the final data cube, regularly sampled in wavelength intervals. The data achieve a $\mathrm{SNR}=5$ at a flux level of $1.0 \times 10^{-17} \mathrm{erg} \mathrm{s}^{-1} \mathrm{~cm}^{-2} \AA^{-1} \operatorname{arcsec}^{-2}$ at $\mathrm{H} \alpha$.

\section{MODELLING THE STELLAR COMPONENT OF THE SPECTRUM}

The WiFeS data allow us to derive the gas kinematics and to map line-ratio diagnostics and dust attenuation across the galaxy system. To achieve this we need a robust estimate of the stellar component of the spectrum which must be identified and subtracted to leave the pure emission-line spectrum. The details of the stellar component modelling and emission-line measurements 
are fully explained elsewhere (see Merluzzi et al. 2013, 2016).

Briefly, we fit the stellar component from the galaxy as a linear combination of 40 simple stellar populations (SSPs) from the Vazdekis et al. (2010) stellar population models covering the full range of stellar ages $(0.06-15 \mathrm{Gyr})$ and three different metallicities $[\mathrm{M} / \mathrm{H}]=-$ $0.41,0.0,+0.22$. The models assume a Kroupa (2001) initial mass function (IMF). They are based on the Medium resolution INT Library of Empirical Spectra of Sanchez-Blazquez et al. (2006), have a nominal resolution of $2.3 \AA$, close to our instrumental resolution, and cover the spectral range $3540-7410 \AA$. The spectra were smoothed spatially (using a $3 \times 3$ spaxel region within the main galaxy body) to achieve a SNR of $\sim 40 / \AA$ for the stellar continuum at $4600-4800 \AA$. For each spaxel, the spatially-smoothed spectrum from the blue arm was fitted with the Vazdekis et al. (2010) models after masking out the regions below $3980 \AA$, which have significantly reduced SNR levels and flux calibration reliability, above $5550 \AA$, where a bright sky line is located, and regions affected emission lines. There were a dozen emission lines that required masking, and for each line we carefully examined the data cube to identify the full range of spectral pixels that are affected by the emission/sky line for at least one spaxel, ensuring that the mask (which is kept fixed for all spaxels) is sufficiently generous to account for the shifts of the emission line due to velocity gradients. We also had to pay particular care to fully mask the broad components linked to the $\mathrm{O}$ III and $\mathrm{H} \beta$ lines for spaxels near the nucleus of the galaxy. For each spaxel, the best-fitting linear combination of SSPs, recession velocity and velocity dispersion to the spatiallysmoothed spectrum is determined, and then renormalized to fit the spectrum from the individual spaxel. This process allows us to subtract the stellar component for spaxels in the outer regions of the galaxy where there is a clear detection of the stellar component, but the SNR is too low to reliably fit complex stellar population models. However, the key aim is to reliably subtract the stellar component to enable robust measurements of the emission lines, in particular for the Balmer lines located in deep absorption features.

In Fig. 5 we show the best-fit linear combination of SSPs in a central region of $6 \times 7$ spaxels in ShaSS 073 . The thick black curve is the original spectrum, the magenta curve is the best-fit to the stellar component and the thicker blue curve is the residual emission. The young $(<1 \mathrm{Gyr})$ component of the stellar population makes up just $4 \%$ of the flux around $4000 \AA$. There is a significant component of $2.5 \mathrm{Gyr}$ old stars, while the remainder is $8-15$ Gyr old.

\section{KINEMATICS OF THE GAS}

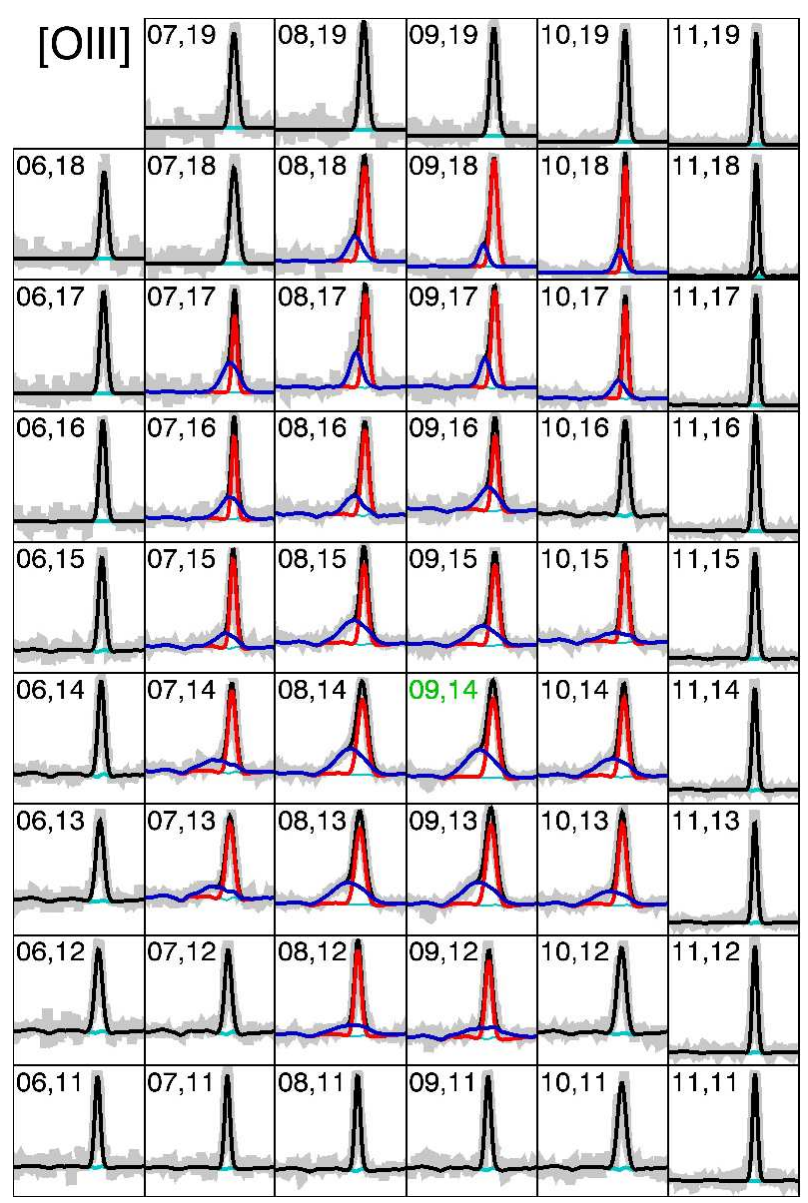

Figure 6. Structure of the [O III $] \lambda 5007$ emission line in a region around the AGN of ShaSS 073. Each panel shows the profile of [O III] in a $1 \times 1 \operatorname{arcsec}^{2}$ spaxel, so that the whole figure covers a region of $6 \times 9 \operatorname{arcsec}^{2}$. Pairs of numbers designate the coordinates in the data-cube, where $(9,14)$, in green, corresponds to the center of ShaSS 073. The curves represent: the observed spectrum (gray), the continuum (cyan), the one-component fit (black) and the two-component fit $($ black $=$ red+blue). Fluxes are scaled to fit the height of the panels.

The gas in the ShaSS 073-622 system is characterized by a complex kinematics. Fig. 6 shows the structure of the [O III] $\lambda 5007$ emission line in a region of $6 \times 9$ spaxels around the center of ShaSS $073^{5}$. Each spaxel is $1 \times 1 \operatorname{arcsec}^{2}$ wide $\left(0.96 \times 0.96 \mathrm{kpc}^{2}\right.$ in projection). The spatial coordinates in the data-cube are indicated, with those of the spaxel corresponding to the center of the main galaxy (i.e. $(9,14)$ ) written in green. The spectra are plotted in gray, while the black-only or black-redblue curves are the fits with one or two spectral components respectively. Emission lines were fitted using the package LZIFU (Ho et al. 2016).

In a number $(\mathrm{N}=24)$ of spaxels the $[\mathrm{O}$ III] line presents

\footnotetext{
${ }^{5}$ We study the kinematics in the [O III] $\lambda 5007$ line because of its brightness. We carefully verified that the kinematics in the other lines $($ e.g. $\mathrm{H} \alpha)$ is fully consistent with the [O III] line.
} 

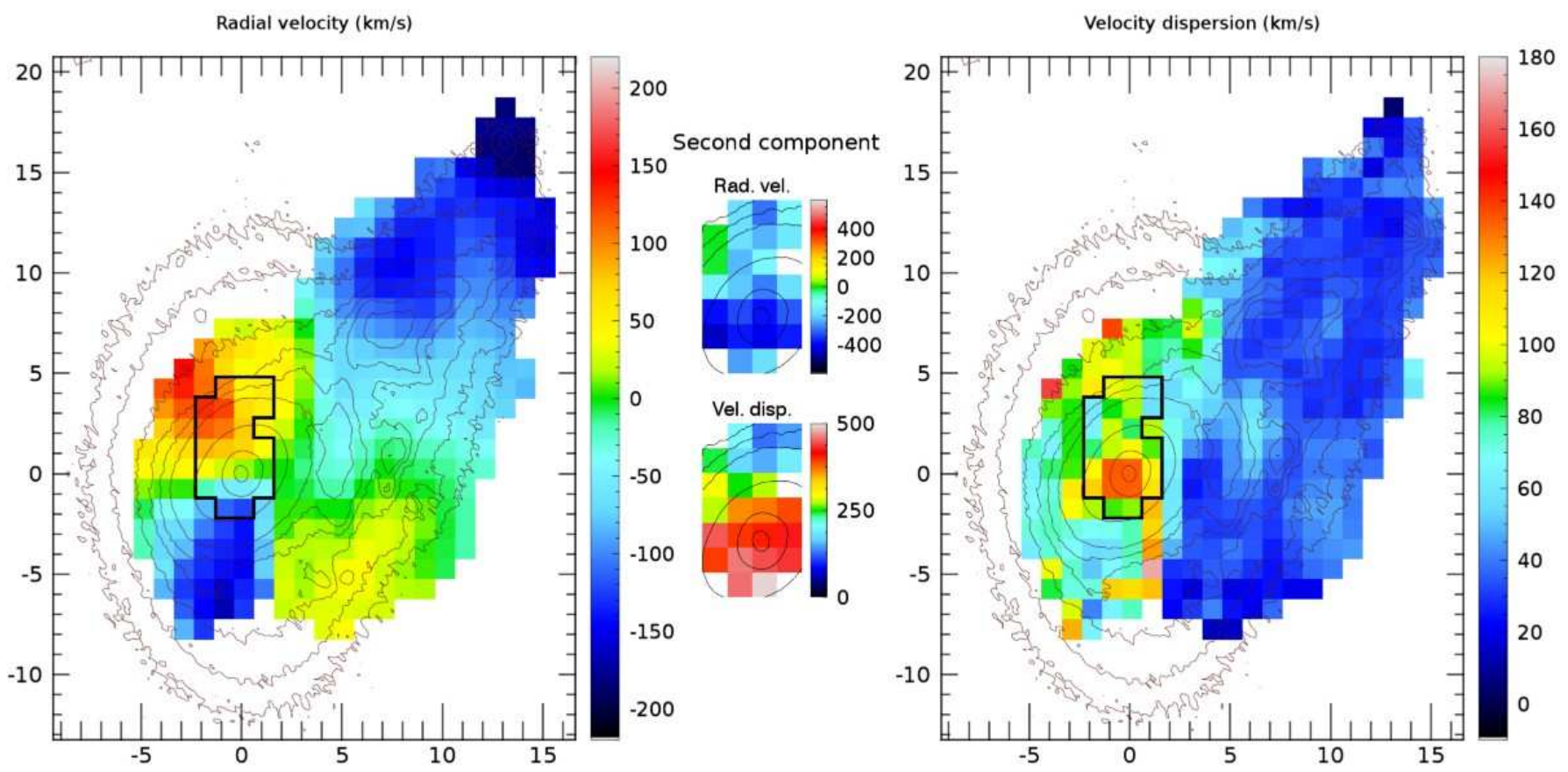

Figure 7. Gas velocity fields (radial velocity and velocity dispersion) of the ShaSS 073-622 system in the [O III] line. The fields are shown for both kinematic components (see text and Fig. 6). The main plots show the kinematics of the 'main component', while the insets are for the second component detected in the area marked by the black polygon. Notice the significant differences in the scales relative to the two components. The contours show the $r$-band brightness distribution. The ticks on the plots mark the distance from the center of the main galaxy in arcseconds. Typical uncertainties are $\sim 10 \mathrm{~km} \mathrm{~s}^{-1}$ for $\mathrm{V}$ and $\sim 10-15 \mathrm{~km} \mathrm{~s} \mathrm{~s}^{-1}$ for $\sigma$.

two kinematically distinct components: one component (plotted in red) has width and redshift values forming a continuous distribution with the rest of the galaxy; the other component (blue) is blue-shifted and in general much wider than the former. We will refer them as main and second components respectively. The statistical significance of decomposition in spectral fitting has been extensively discussed in the literature (e.g. Ho et al. 2016). In a first instance, we applied the likelihood ratio test to our data (Eq. 1 in Ho et al. 2016), obtaining however mixed results, in some cases in obvious contradiction with reality. We thus decided to select the spectra with double components based on visual inspection.

The gas velocity fields are shown in Fig. 7. Within the dominant galaxy the kinematic fields have fairly smooth trends, especially in radial velocity $\left(\mathrm{V}_{r}\right)$. The second component, shown in the insets, is systematically blueshifted and has high velocity dispersion.

\subsection{Kinematics of the main gas component}

The radial velocity field of the main component gas is fairly consistent with rotation of the gas in the disks of both galaxies. In the main galaxy, the signature of an orderly rotational motion is clearly visible in the eastern side of its disk (where there is no superposition of the
$[\mathrm{NII}] /(\mathrm{H} \alpha)$

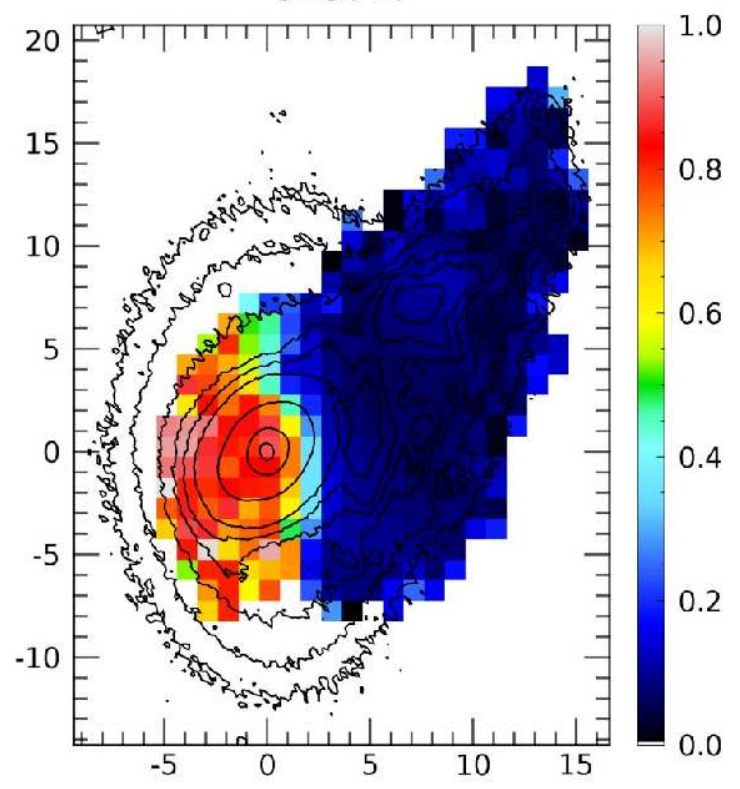

Figure 8. The $[\mathrm{N} \mathrm{II}] / \mathrm{H} \alpha$ line ratio which sharply separates the two galaxies.

two galaxies). In ShaSS 622 the rotation in the opposite sense to the main galaxy is visible along its whole extent. The survival of such an ordered rotation is clear evidence that the interaction between the two galaxies 
is at its starting phase. The kinematics of ShaSS 622 will be further considered in Sect. 8 .

To better understand the situation in the region of superposition of the two galaxies, we show in Fig. 8 the distribution of the $[\mathrm{N} \mathrm{II}] / \mathrm{H} \alpha$ line ratio, which is a proxy of gas metallicity. We note that in ShaSS $622[\mathrm{~N} \mathrm{II}] / \mathrm{H} \alpha$ is always lower than $\sim 0.2$, while in the main galaxy it is generally higher than $\sim 0.6$, with a very narrow $(\sim 1-$ 2 arcsec-wide) 'transition' strip, which is likely the superposition of the two sets of emission. Thus, this flux ratio effectively separates the gas of the two galaxies. A 'transition strip' is clearly seen also in the radial velocity field, with values of $\mathrm{V}_{r}$ intermediate between those of the two disks.

The separation between the two gas disk systems is also evident from the distribution of the velocity dispersions, which are much lower ( $\sim 40 \mathrm{~km} \mathrm{~s}^{-1}$ on average) in ShaSS 622 than in the main galaxy $\left(\sim 60-170 \mathrm{~km} \mathrm{~s}^{-1}\right)$. The mixing strip is characterised by a local increase of $\sigma$ (the absolute maximum of $\sigma$ of $170 \mathrm{~km} \mathrm{~s}^{-1}$ actually is located in the southern strip), likely due to the superposition of the motions of the gas in the two disks. A maximum velocity dispersion of $135 \mathrm{~km} \mathrm{~s}^{-1}$ is located in the center of the main galaxy. The nucleus of ShaSS 622 is blue-shifted with respect to that of ShaSS 073 by $\sim 130 \mathrm{~km} \mathrm{~s}^{-1}$, which should represent the component along the line of sight of the relative systemic motion of the two galaxies.

\subsection{Kinematics of the second gas component}

The second gas component is generally blue-shifted with respect to the disk of ShaSS 073 and has also a higher velocity dispersion. The value of $\sigma$ clearly divides the second component in two distinct areas: the circum-nuclear $(r \lesssim 2-3 \mathrm{kpc})$ region with $\sigma>360 \mathrm{~km} \mathrm{~s}^{-1}$ reaching $490 \mathrm{~km} \mathrm{~s}^{-1}$ (red-white colors in the lower inset of Fig. 7) and radial velocity down to $-440 \mathrm{~km} \mathrm{~s}^{-1}$ and a northern area with more moderate values. We anticipate that this separation based on kinematics corresponds to distinct physical properties of the gas, as will be shown in the following Sections, and will postpone the discussion of the lower- $\sigma$ component.

The kinematics of the circum-nuclear gas, i.e. large negative radial velocities accompanied with large velocity dispersion within a scale of a few kiloparsecs, is typical of AGN outflows (e.g. Fischer et al. 2013; Karouzos et al. 2016). Outflows are quite common in type 2 AGNs (e.g. Harrison et al. 2014; McElroy et al. 2015; Woo et al. 2016), and generally have the structure of a hollow bicone in which the gas flows in the external corona, while the AGN radiation escapes from the inner cone (e.g. Crenshaw et al. 2000; Fischer et al. 2013). In general, only the blue-shifted half of the outflow is observed (as in our case) because the other part is obscured by the

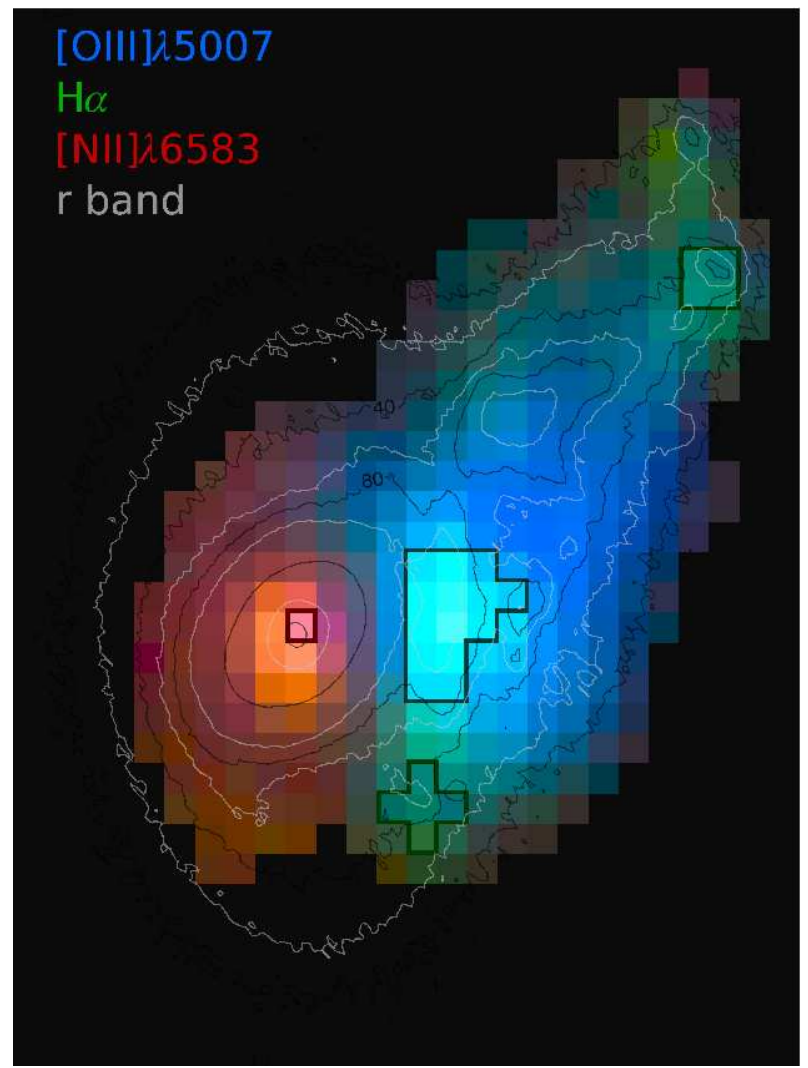

Figure 9. A composite RGB image derived from the fluxes in the emission lines [N II] $\lambda 6583(\mathrm{R}), \mathrm{H} \alpha(\mathrm{G})$, and [O III] $\lambda 5007$ (B), with isophotes from the VST $r$-band image superimposed. The black polygons mark the groups of spaxels whose spectra were combined to produce the representative spectra shown in Figs. 16 and 17. From top to bottom and on the right: HII Region \# 1, High-Excitation Region, HII Region \# 2. On the center left: $A G N$.

dust in the galactic disk. The limited spatial resolution of our IFS prevents a detailed study of the structure of the outflow (e.g. using the models of Fisher et al. 2013 or Bae \& Woo 2016). We will however be able to gain insight into the geometry of the ionization cone using the properties of the disk region of ShaSS 622 affected by it (see Sect. 8).

\section{PHYSICAL PROPERTIES OF THE GAS}

Fig. 9 shows a RGB composite image derived from the fluxes in [N II] $\lambda 6583$ (red), $\mathrm{H} \alpha$ (green), and [O III] $\lambda 5007$ (blue). The ShaSS 073-622 complex appears to be clearly divided in three regions in this figure. The main galaxy is seen very strongly in the $[\mathrm{N}$ II $] \lambda 6583$ line, indicating a high chemical abundance, probably along with a high reddening (see below). Not surprising for an AGN, the nucleus is very strong in this line. The green areas at the extremes of the disk of ShaSS 622 are constituted by individual $\mathrm{HII}$ region complexes, as is apparent comparing the positions of the isophotes with the images in Fig. 1.

As already noted, the most striking emission feature 
$[\mathrm{OIII}] /(\mathrm{H} \alpha / 2.86)$

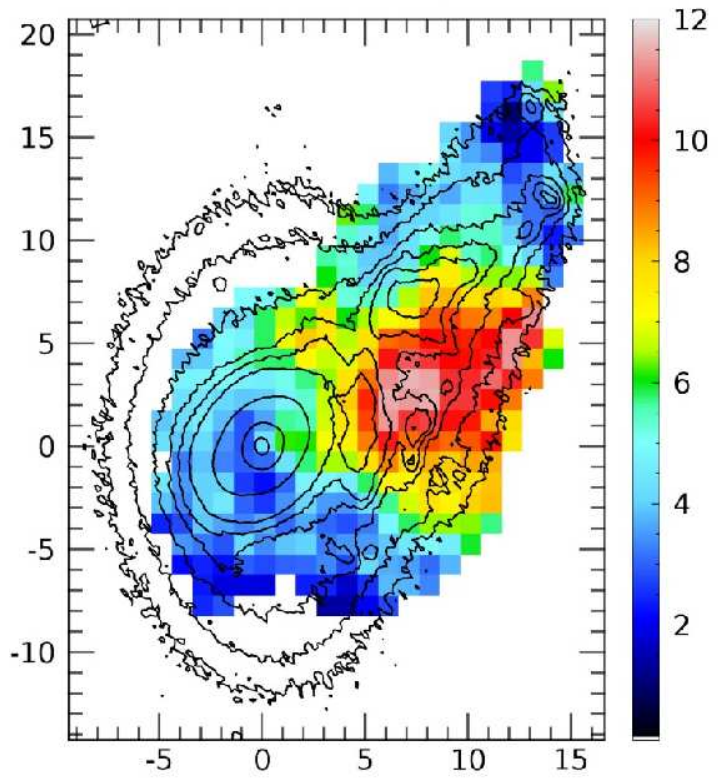

Figure 10. The 'effective' $[\mathrm{O}$ III $] / \mathrm{H} \beta$ line ratio (derived from the reddening uncorrected [O III] and $\mathrm{H} \alpha$ line flux maps). Note the highly excited region in the disk of ShaSS 622.

of ShaSS 622 is the region of very strong [O III] emission (blue in the figure, see also bottom right panel of Fig. 1). The brightest part of this region is the one closest to the nucleus of ShaSS 073. To get a more precise idea on its shape, we show in Fig. 10 the distribution of the 'effective' $[\mathrm{O} I I I] / \mathrm{H} \beta$ line ratio, which can be taken as a proxy for the ionization parameter for regions excited by an AGN (Davies et al. 2016). This is taken from the [O III] $\lambda 5007$ flux distribution (uncorrected for local reddening, which is however very low, cf. Fig. 12) and the $\mathrm{H} \alpha$ flux map (likewise uncorrected for reddening) divided by the standard Balmer ratio of 2.86 , which provides an approximate $\mathrm{H} \beta$ flux from the measured $\mathrm{H} \alpha$. Fig. 10 reveals the presence of highly ionized gas in a well-defined area of the ShaSS 622 disk. The border of this area appears to be rather sharp and bears some resemblance with a parabola. Considering that the AGN outflow was already identified in Sect. 5.2, we associate this high-excitation area with the intersection of the ionization cone from the AGN with the gas of ShaSS 622 and we will refer to it as "the high excitation region" (HER). The aperture and orientation of the ionization cone will be determined in Sect. 8 .

A wealth of physical and chemical properties of the ionized gas can be derived from the ratios of emissionline fluxes. In a previous work (Merluzzi et al. 2013) we have shown that to obtain flux ratios with uncertainties lower than $\sim 30 \%$, the individual lines must have a signal-to-noise ratio (SNR) $\gtrsim 20$. To achieve these SNRs we spatially binned the data by means of the 'Weighted Voronoi Tessellation' (WVT) method de-

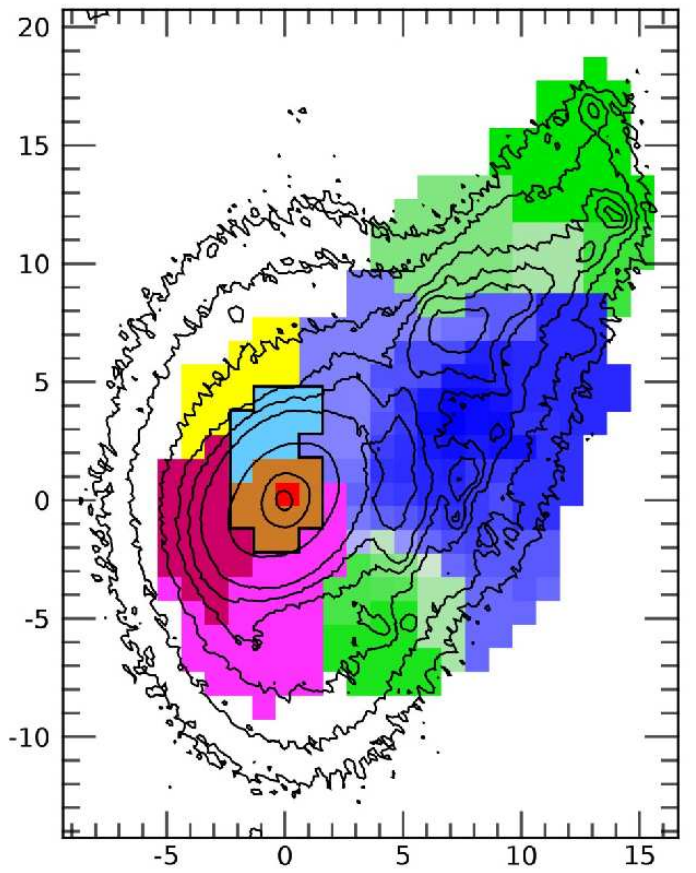

Figure 11. Identification of the 73 regions in which the spectra have been combined to derive the line ratios used in our analysis. The region with a double gas component is marked with a black polygon. The rationale for the choice of colors is explained in the text and becomes clear in Fig. 13.

scribed by Diehl \& Statler $^{6}$ (2006). This attempts to reach a fixed target SNR in all bins (see Merluzzi et al. 2013, for details). After some tests, we set as our target $\mathrm{SNR}=150$ for the $\mathrm{H} \alpha$ line. To obtain a spatial mapping consistent with the distribution of the properties of the gas outlined above, we applied the WVT to areas clearly associated with either one or the other galaxy.

The 73 regions identified in this way are shown in Fig. 11. The regions belonging to ShaSS 622 are colorcoded according their $[\mathrm{O}$ III $] / \mathrm{H} \beta$ decreasing ratios from blue to green. ShaSS 073 was divided in six regions: three in the disk, one in the center (red) and two including the other spaxels with two-component gas (orange and cyan). The orange region is where the second component has velocity dispersion $>360 \mathrm{~km} \mathrm{~s}^{-1}$ and is more blue-shifted (corresponding to the gas in the outflow), while in the cyan area these features of the second component are much less prominent (cf. Sec. 5.2). The colors for ShaSS 073 were chosen for visual convenience only.

\subsection{Dust attenuation}

Fig. 12 shows the distribution of the dust attenuation derived from the $\mathrm{H} \alpha / \mathrm{H} \beta$ line ratio, in terms of the visual

${ }^{6}$ http://www.phy.ohiou.edu/diehl/WVT 


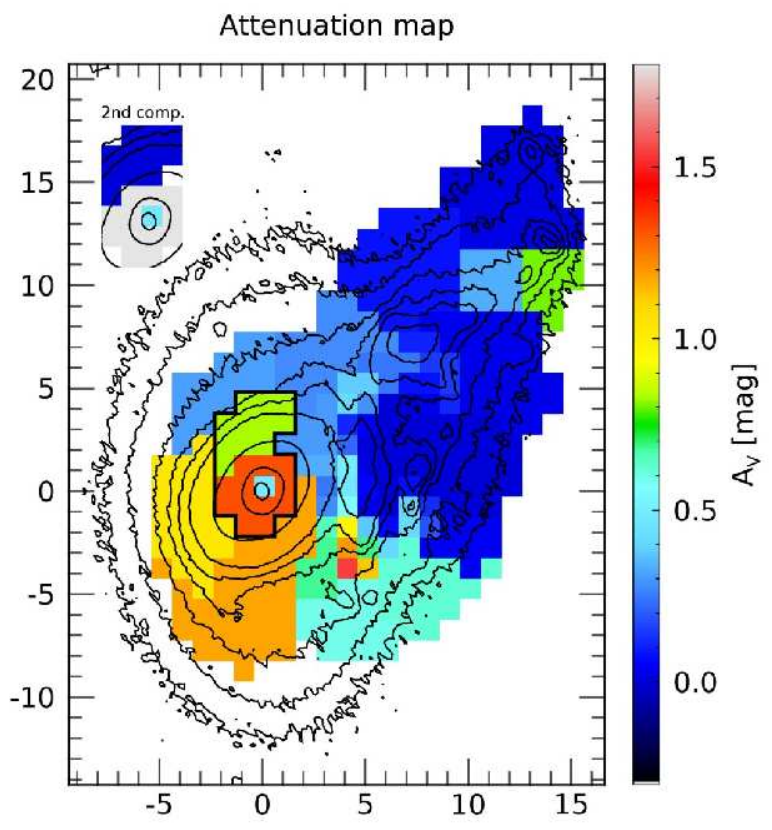

Figure 12. Attenuation map ( $A_{V}$ magnitudes) derived as described in the text. The area where two spectral components are present is marked by the black polygon. The attenuation in the outflow is shown in the inset and reaches $A_{V}=3$ (light gray area).

extinction $A_{V}$, derived in the binned regions of Fig. 11. We use the theoretical attenuation curve by Fischera \& Dopita (2005) with $\mathrm{R}_{V}=4.5$. To derive the attenuation map we adopted an $\mathrm{H} \alpha / \mathrm{H} \beta$ ratio of 2.86 . The uncertainties on $A_{V}$ are in the range 0.1-0.3 mag over ShaSS 622 and reach $0.5-1.1 \mathrm{mag}$ in the eastern disk of ShaSS 073, and in the outflow, due to lower SNR of the $\mathrm{H} \beta$ line.

The attenuation is generally low $(<0.5 \mathrm{mag})$ in ShaSS 622. It is somewhat higher in a limited area in the NW disk $\left(A_{V} \sim 0.7\right)$, and in the area where the SE disk of ShaSS 622 and the SW disk of ShaSS 073 overlap $\left(A_{V} \sim 0.6\right)$. The highest dust extinction takes place in the central and SE disk of ShaSS 073 and in the broad-line part of the second component, where it reaches $A_{V} \sim 3 \pm 1$ magnitudes. A local minimum of $A_{V} \sim 0.5 \pm 0.6$ is observed in the nucleus of ShaSS 073 in both the narrow and broad components. This fact is consistent with either dust removal driven by radiation pressure or dust destruction from the UV radiation of the AGN.

Overall, the dust attenuation follows the general trend of the properties of the gas, being fairly distinct between the two galaxies. The dust is clearly more abundant in the disk of ShaSS 073 than in the companion.

\subsection{Line ratio diagnostics}

To study the variation in excitation and the mode of excitation of the ionized gas in both galaxies we use the BPT emission line ratio diagrams Baldwin et al.
(1981) as refined by Veilleux \& Osterbrock (1987), Kewley et al. (2001), Kauffmann et al. (2003) and Kewley et al. (2006).

In Fig. 13 we show the measured line ratios for the different galaxy regions. The red curves mark the computed upper limit to star-forming regions defined by Kewley et al. (2001), while the dashed blue curve in the left panel marks the empirical upper limit for $\mathrm{H}$ II regions from Kauffmann et al. (2003). Between the red curve and the dashed blue curve, 'composite' systems are found whose emission may be characterised by a mixture of AGN and HII regions or by shock excitation. The blue continuous line in the central and right panels divides the line ratios typical of Seyfert galaxies (upper part of the diagram), from those of low ionization nuclear emission line regions (LINERs).

The regions of our system separate into distinct groups: those where the gas tends to be mainly photoionized by star formation (green dots), the HER (blue dots) which reach high levels of excitation $(\log ([\mathrm{O} \mathrm{III}] /$ $\mathrm{H} \beta) \sim 1$ ). The third group includes all the regions of ShaSS 073. For the three regions populated by twocomponent gas (colors red, orange and cyan in Fig. 13), square symbols are for the main component and triangles represent the second component.

The gas in ShaSS 073 is generally characterized by a lower excitation than the HER $(\log ([\mathrm{O} \mathrm{III}] / \mathrm{H} \beta) \lesssim 0.75)$, with the notable exception of the gas in the outflow (red and orange triangles). The second gas component in the northern disk (cyan triangle) is instead mixed with the rest of the gas of ShaSS 622 in the diagnostic diagrams, showing that it belongs to the disk population and has therefore a different nature from the outflow (see Sect. 5.2). This will be further discussed in Sect. 8 .

\section{PHOTOIONIZATION MODELLING}

\subsection{Analysis using Diagnostic Diagrams}

A better insight into the physics of the different regions can be obtained by comparing the observations explicitly with the results of photoionization modelling. In Fig. 14 we have used the theoretical AGN grid of models from Davies et al. (2016) to analyse both the AGN and the region within the HER of ShaSS 622. These models were constructed using the Mappings 5.0 code $^{7}$ (Sutherland et al. 2017, in prep.), and use the Local Galactic Concordance abundance scaling described by Nicholls et al. (2017). The input spectrum for the AGN is kept fixed in this grid.

Fig. 14 makes the abundance difference between the AGN (red dots) and the HER (blue dots) in the com-

\footnotetext{
${ }^{7}$ Available at miocene.anu.edu.au/Mappings
} 


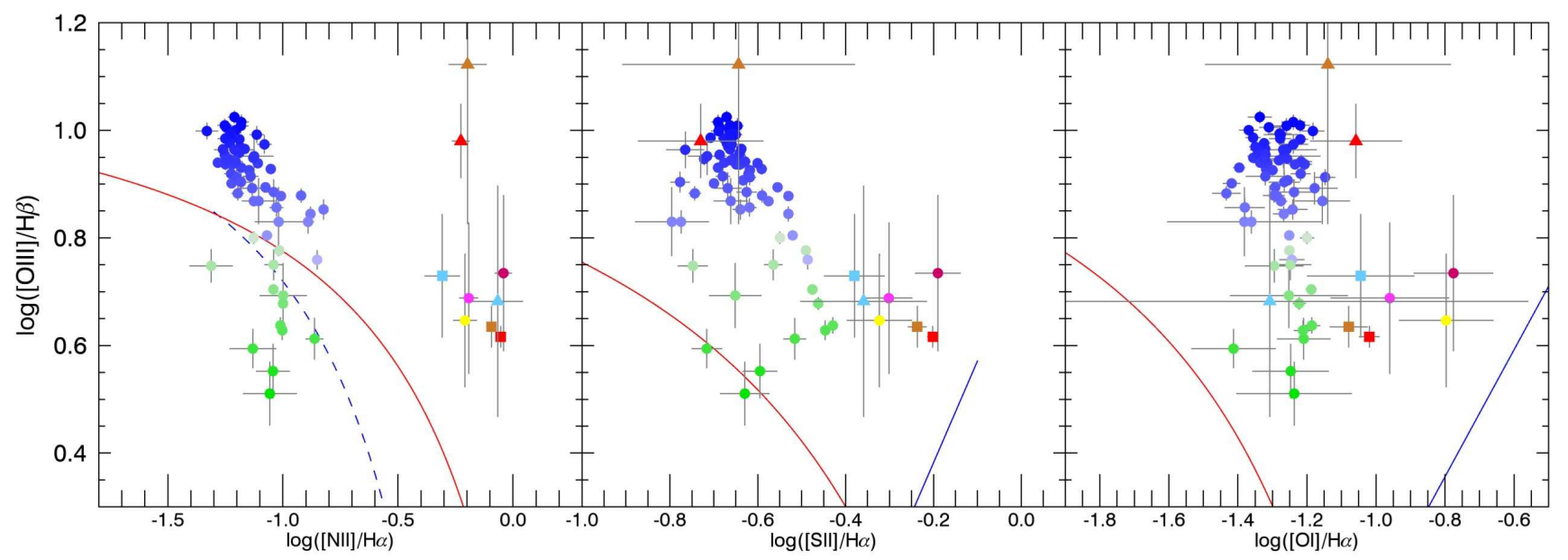

Figure 13. Line ratios diagnostic diagrams of the different regions of the ShaSS 073-622 system. The curves are the empirical (blue dashed) and theoretical (red continuum) upper limits for H II regions, while the blue straight line divides Seyfert galaxies from LINERs (upper and lower part respectively). The colors correspond to Fig. 11. For the three regions populated by two-component gas (with colors red, orange and cyan), square symbols are for the main component and triangles represent the second component.

panion galaxy very clear. The AGN is fitted with a $\sim 1.5$ times super-solar abundance while in the HER is $\sim 0.4$ solar. The ionization parameter, $\log U$ in the AGN is typically -2.7 , while in the outflow much higher values ( $\log U \sim-1)$ are indicated. For the HER, the ionization parameter reaches -1.8 , falling to -2.5 at the edge of the AGN-ionized region.

The analysis of the 'normal' star-forming regions of ShaSS 622 (the green bins in Fig. 11) requires that we compare these with $\mathrm{H}$ II region models. This is shown in Fig. 15. For these we use the same approach as described by Dopita et al. (2013) with input EUV spectra derived from the Starburst 99 code (Leitherer et al. 1999). The grid shown is for a high pressure ISM with $\log (P / k)=6.8\left(\mathrm{~cm}^{-3} \mathrm{~K}\right)$.

In Fig. 15, it is evident that the $\mathrm{H}$ II regions in the $[\mathrm{N} \mathrm{II}] / \mathrm{H} \alpha$ diagnostic are consistent with a metallicity of $0.3-0.7$ solar and a high ionization parameter. However, the $[\mathrm{S} \mathrm{II}] / \mathrm{H} \alpha$ diagnostic has the observed points lying off the grid. Both these facts, and the high ionization parameter implied by the $[\mathrm{N} \mathrm{II}] / \mathrm{H} \alpha$ diagnostic could be explained by contamination of the bins lying outside the main ionization cone by AGN photons penetrating the diffuse ISM of ShaSS 622 .

The parameters of photoionized regions derived from BPT diagrams alone are limited by the presence of other factors influencing both the position and shape of the theoretical grids. These include the gas pressure $\log (P / k)\left(\mathrm{cm}^{-3} \mathrm{~K}\right)$, the depletion factor of iron and other elements onto grains, and the details of the shape of the EUV spectrum.

In order to facilitate a more detailed photoioniza- tion analysis of the AGN in the main galaxy, the highexcitation region of the companion galaxy, and the two brightest $\mathrm{H}$ II regions in the companion galaxy, we extracted spectra summing over the spaxels indicated in Fig. 9. The resultant spectra are plotted in Figs. 16 and 17. H II region \#1 corresponds to the northern H II region in the companion galaxy, and $\mathrm{H} I$ I region $\# 2$ is the southern object in Fig. 9.

In Fig. 16, the HER (top spectrum) is characterised by the presence of very high-excitation species such as $[\mathrm{Ne} \mathrm{V}]$ and [Fe VII]. However, the relative ratios of the $[\mathrm{OI}],[\mathrm{NII}]$ and $[\mathrm{SII}]$ lines in the red, and as already noted above the $[\mathrm{N}$ II $] / \mathrm{H} \alpha$ ratio implies that the chemical abundance is appreciably sub-solar. As such this spectrum is quite unlike what is usually seen in the ENLR of local Seyfert-2 galaxies, which appear to have supersolar metallicities (Davies et al. 2016).

The spectrum of the nucleus of the main galaxy is that of a Seyfert-2 with typically strong [O III] emission with respect to $\mathrm{H} \beta$, and strong [N $\mathrm{NI}]$ emission with respect to $\mathrm{H} \alpha$. Also [S II] and [OI] are both fairly strong. A relatively strong continuum of old stars is also present.

\subsection{Bayesian Photoionization Analysis}

As noted above, the theoretical grid of models is at least a 5-D space: chemical abundances, ionization parameter, gas pressure, dust depletion factors, and the shape of the EUV spectrum (which may itself be characterised by more than one variable). In order to objectively locate the position of an observed object in this space we require a Bayesian approach to the problem.

In this section we apply the new package NebulaBayes, 

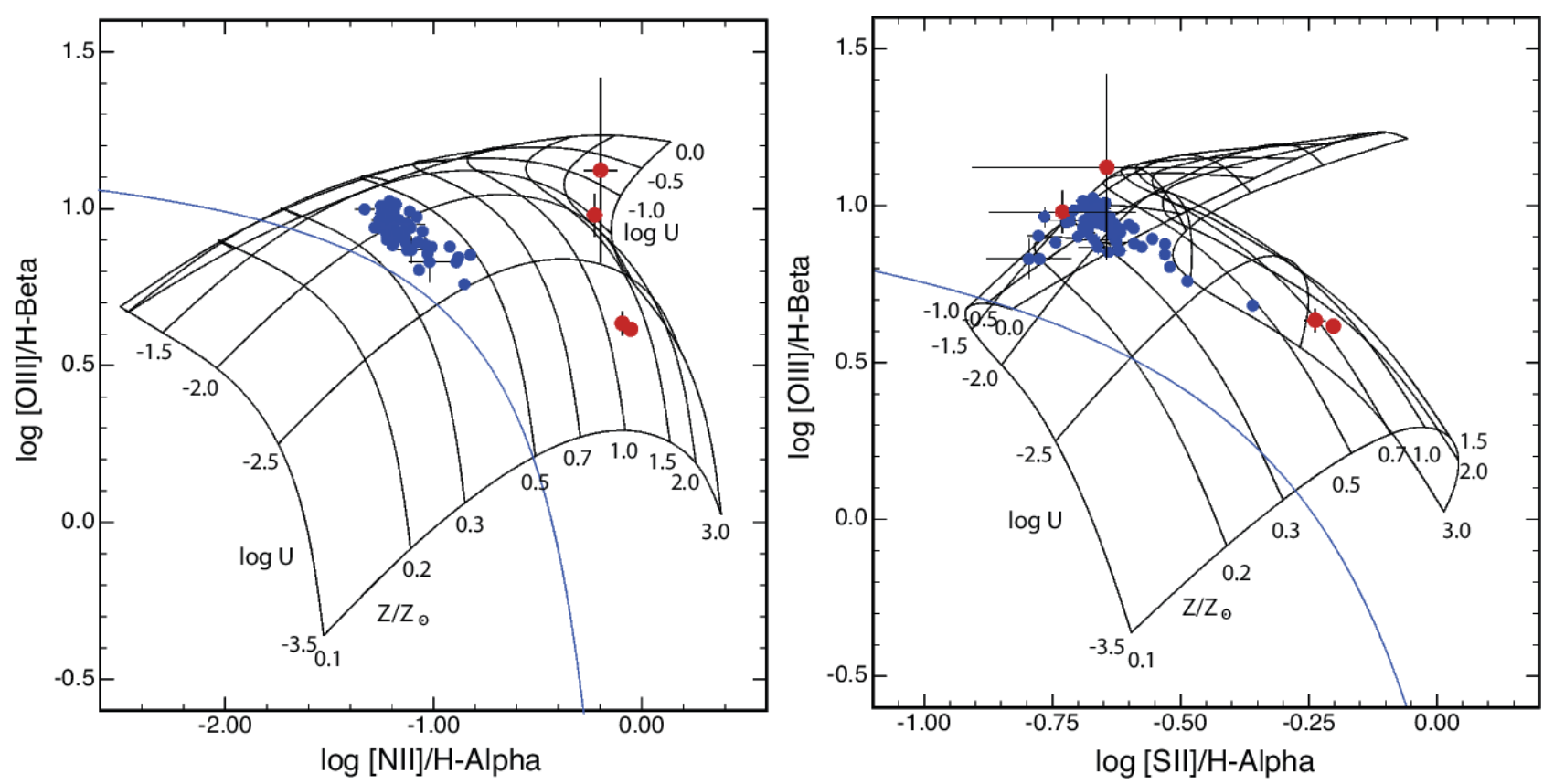

Figure 14. The theoretical AGN grid of models from Davies et al. (2016) compared with the observations of the HER (blue dots) and the region around the AGN in ShaSS 073 (red dots).
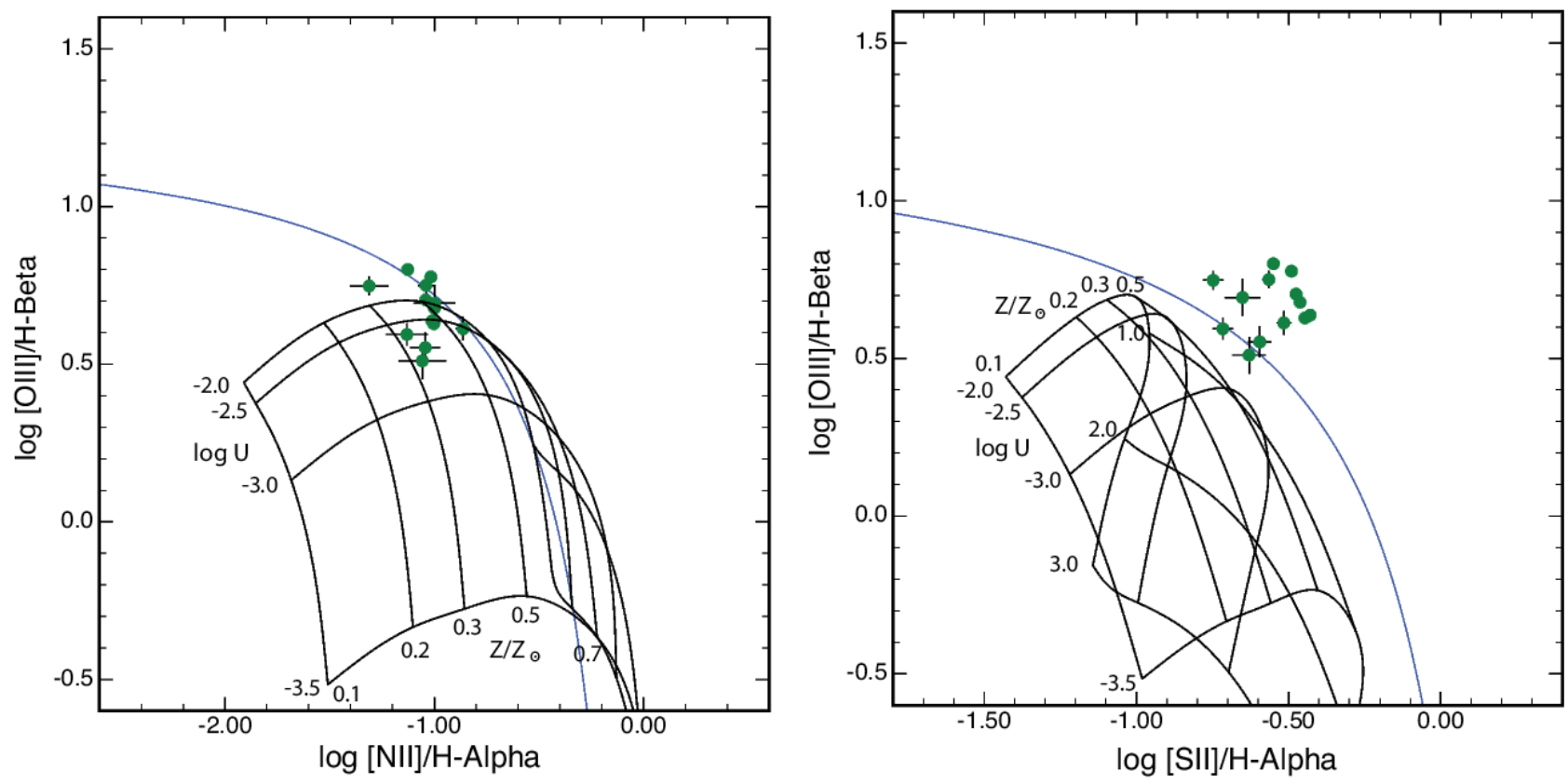

Figure 15. The theoretical grid of HII region models from Davies et al. (2016) compared with the observations of the regions of ShaSS 622 shown in green in Fig. 11. The low metallicity inferred for the HER in Fig. 14 is confirmed in these star formation regions lying outside the ionization cone. 


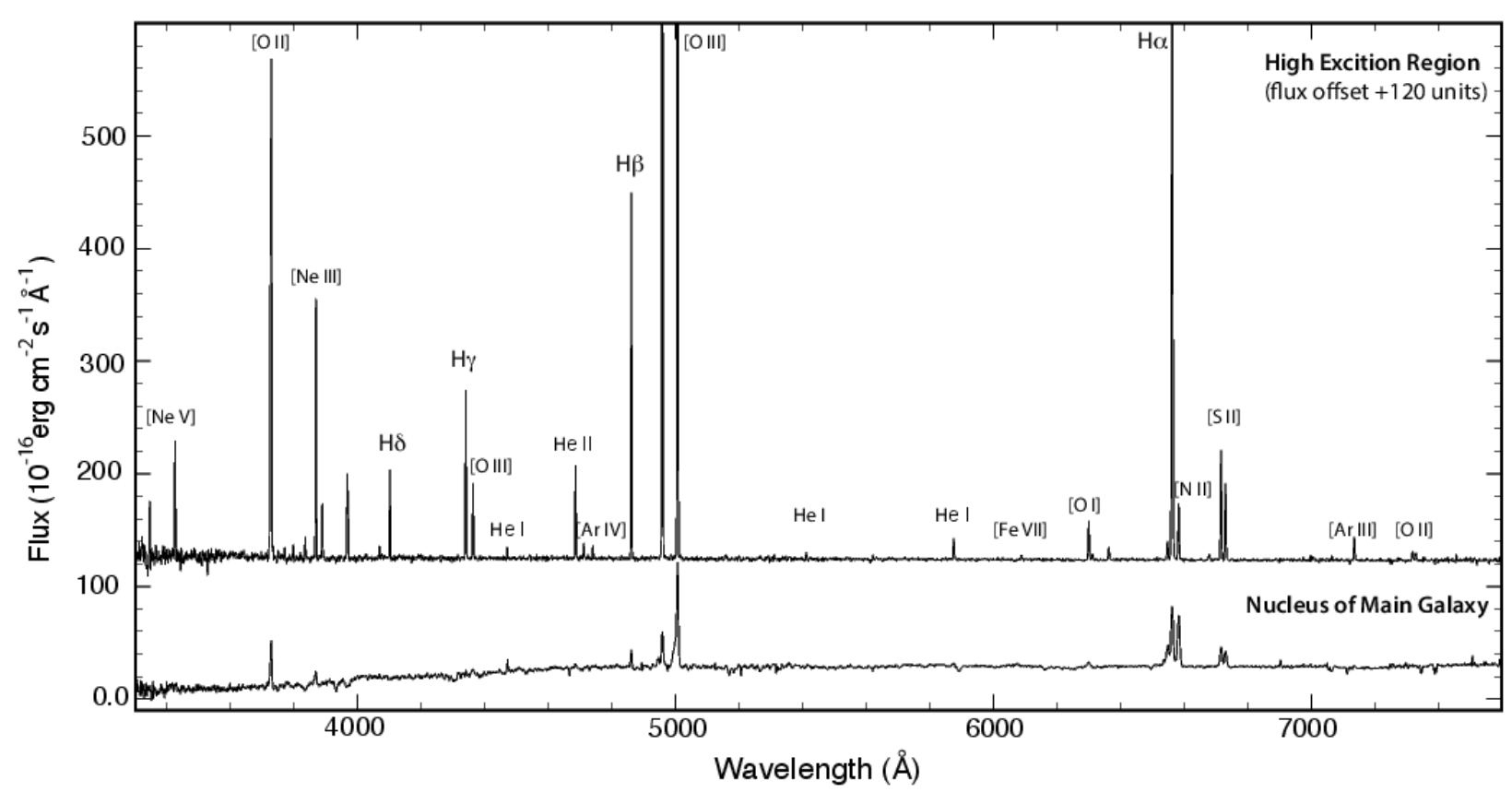

Figure 16. The extracted spectra of the nuclear region of the main galaxy and the HER. The latter spectrum has been scaled to show the fainter lines, so both $\mathrm{H} \alpha$ and the [O III] lines are off scale. Note the presence of highly excited species such as [Ne $\mathrm{V}]$ and [Fe VII]. The spectrum of the nucleus of the main galaxy is that of a Seyfert-2 with a relatively strong continuum of old stars, and is the presumed source of the excitation of the high-excitation region of the companion galaxy.

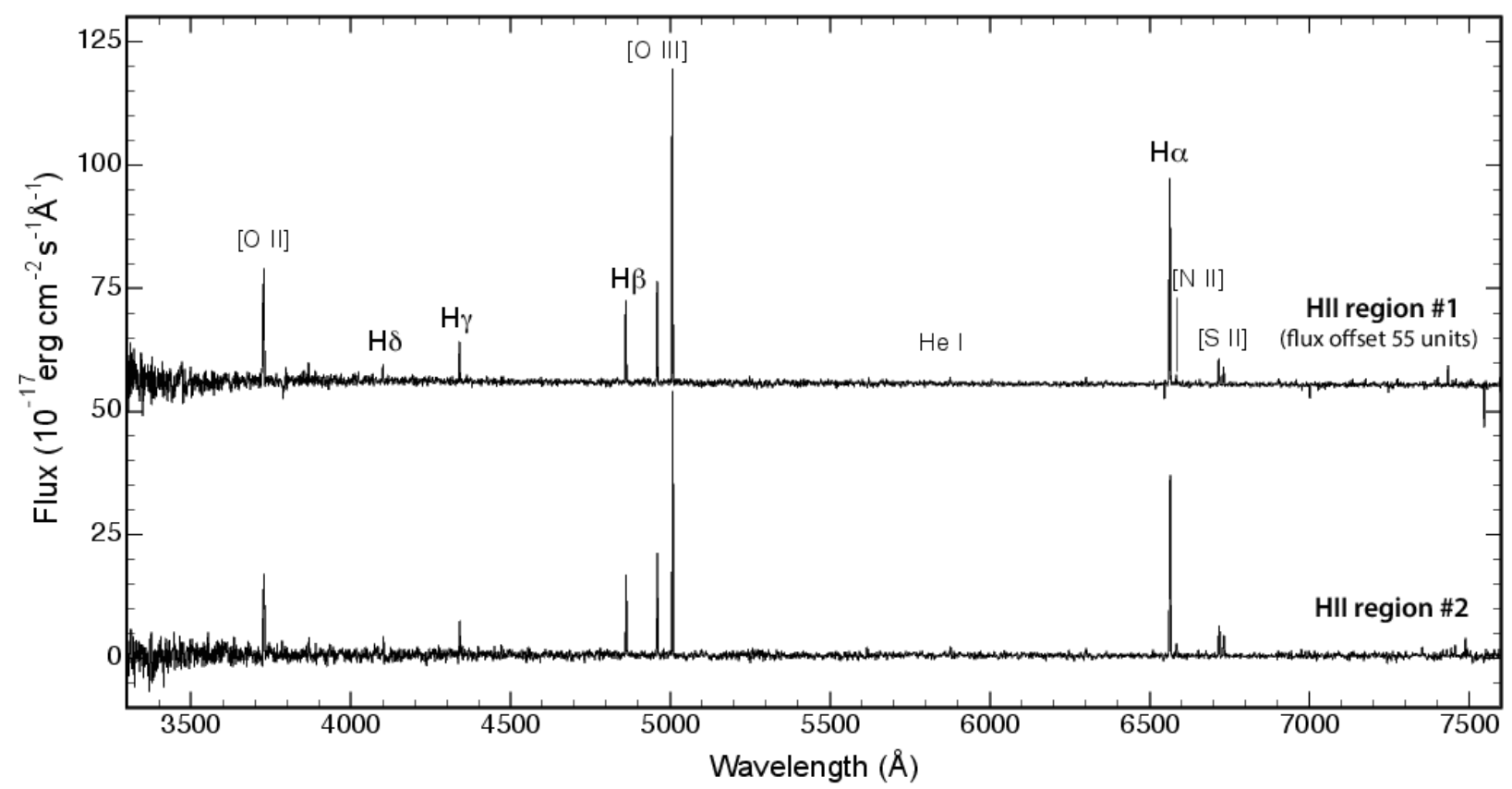

Figure 17. The extracted spectra of the $\mathrm{H}$ II region complexes. The weak $[\mathrm{N} \mathrm{II}] / \mathrm{H} \alpha$ ratio, and the relative strength of $[\mathrm{N}$ II $]$ to [S II] imply that the chemical abundances in these $\mathrm{H}$ II regions are appreciably sub-solar. 

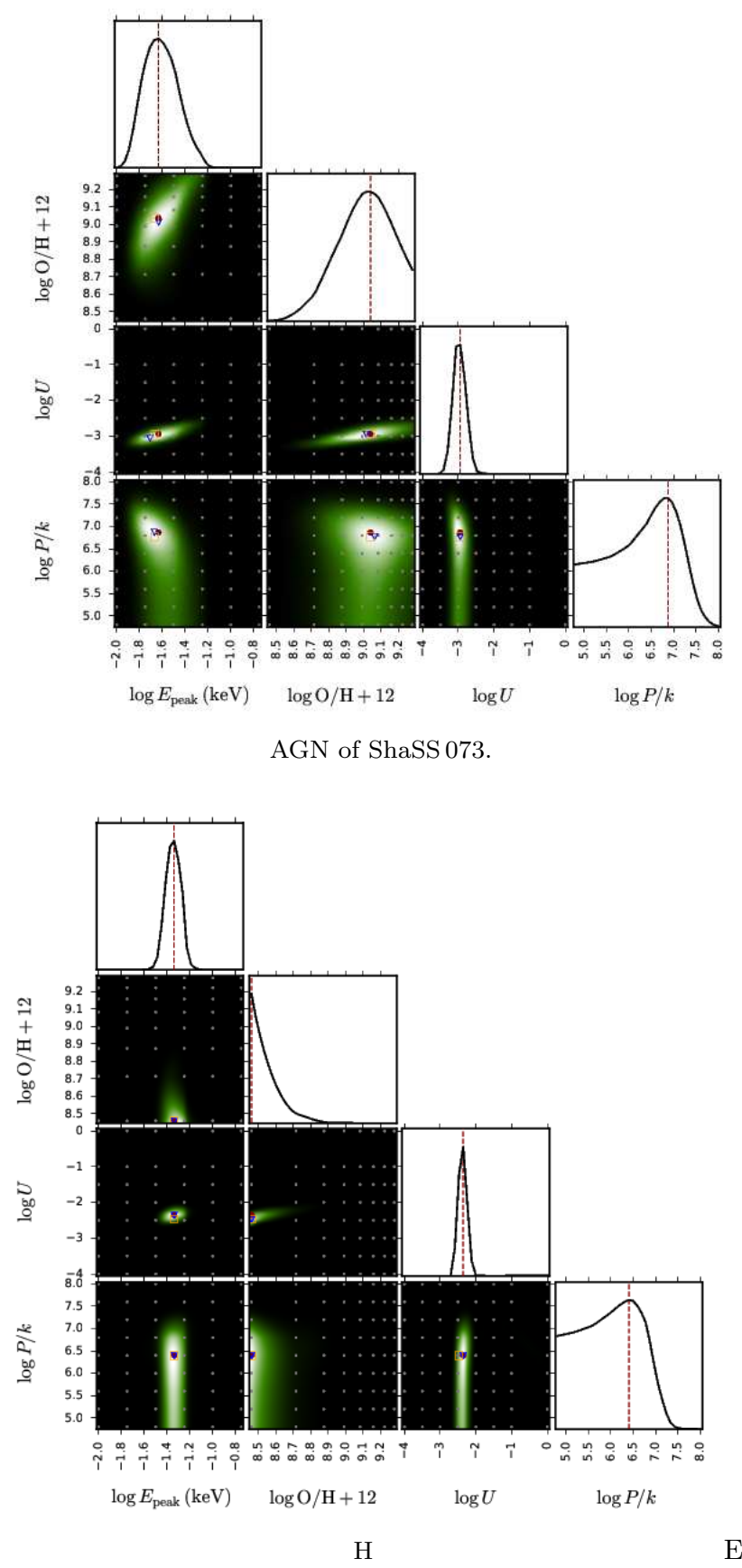

ER

Figure 18. Marginalised posterior distributions showing constraints on the peak energy of the EUV radiation field $E_{\text {peak }}$, the oxygen abundance $\log \mathrm{O} / \mathrm{H}+12$, the ionization parameter $\log U$, and the gas pressure $\log P / k$. Different shades of green (dark to light) indicate probability densities from low to high. Gray dots represent model grid-points. The 1D marginalized posteriors are drawn with black lines, with the brown vertical dotted line at the peak of a $1 \mathrm{D}$ posterior indicating the value taken as the parameter estimate. On each distribution is plotted the model defined by the parameter estimates (brown circle), the peak of the 2D marginalized distribution (blue open triangle) and the projected peak of the full $n$-dimensional PDF (orange open square). a generalization of IZI (Blanc et al. 2015), which constrains nebular parameters using a novel Bayesian approach. Whereas IZI works in two dimensions, constraining only the ionization parameter and metallicity, NebulaBayes works in arbitrary dimensions and is parameter agnostic. The method compares observed line fluxes to an extensive grid of pre-computed photoionization models, thereby calculating the relative probabilities of the model parameters at all points in the multiparameter space. NebulaBayes will be described in more detail and made available to the community in the near future (Thomas et al., in preparation).

The computed $\mathrm{H}$ II region grid has three parameters the metallicity $12+\log \mathrm{O} / \mathrm{H}$, the ionization parameter $\log U$, and the gas pressure $\log P / k$. The logarithmic dust depletion factor is kept fixed at -1.5 for Fe. For the input EUV spectrum of $\mathrm{H}$ II regions we use the spectra described by Dopita et al. (2013) which are derived from the Starburst 99 code (Leitherer et al. 1999).

The NLR photoionization grid has metallicity $12+$ $\log \mathrm{O} / \mathrm{H}$, ionization parameter $\log U$, and gas pressure $\log P / k$ plus a fourth parameter, which describes the peak energy of the accretion disk emission $E_{\text {peak }}$ (measured in $\left.\log _{10} E / \mathrm{keV}\right)$. This fourth parameter derives from the AGN ionizing continuum model OXAF (Thomas et al. 2016) which is used in the MAPPINGS modelling.

The computational approach is as follows: Firstly, a prior is defined over the parameter space to include prior expectations of parameter values in the Bayesian parameter inference. Secondly, the likelihood (the probability of the data given the model, i.e. given each set of parameters in the parameter space) is calculated assuming Gaussian errors on the observed fluxes and weighting all emission lines equally. The posterior (which is the probability of the model given the data, and describes the relative probabilities of sets of parameters in the parameter space) is then calculated according to Bayes Theorem by multiplying the prior and the likelihood. Parameter estimates and credible intervals are obtained by marginalizing ${ }^{8}$ the posterior probability distribution function (PDF) to obtain 1D PDFs for each nebular parameter.

\footnotetext{
${ }^{8}$ If $N$ is the number of parameters, this means to integrate over $N-n$ dimensions to obtain the $n$-dimensional distribution for $n$ parameters.
} 
Table 2. NebulaBayes parameter estimates with $68 \%$ confidence intervals.

\begin{tabular}{lcccc}
\hline \hline Region & H II \#1 & H II \#2 & Ion. cone & AGN \\
& & & & \\
\hline $12+\log \mathrm{O} / \mathrm{H}$ & $8.4 \pm 0.1$ & $8.6 \pm 0.1$ & $<8.5$ & $9.0 \pm 0.2$ \\
\hline $\log (P / k)$ & $6.5_{-2.0}^{+0.1}$ & $6.5_{-1.8}^{+0.1}$ & $6.4_{-1.2}^{+0.3}$ & $6.9_{-1.5}^{+0.3}$ \\
\hline $\log U$ & $-3.1 \pm 0.1$ & $-3.1 \pm 0.1$ & $-2.4 \pm 0.2$ & $-3.0_{-0.2}^{+0.3}$ \\
\hline $\log \left(E_{\text {peak }} / \mathrm{keV}\right)$ & - & - & $-1.3 \pm 0.1$ & $-1.6 \pm 0.2$ \\
\hline
\end{tabular}

The $[\mathrm{S} \mathrm{II}] \lambda \lambda 6731 / 6717$ ratio is the main prior used, since this directly constrains the gas pressure. For the HER material, the excitation as derived from the He II $\lambda 4686 /$ He I $\lambda 5876$ ratio was also used as a prior. For these priors, the observed line ratio is compared to a predicted ratio and a probability is obtained by taking into account the observational errors on the observed ratio. This calculation at every grid point in the parameter space yields an $n$-dimensional PDF. The PDFs for each line ratio prior are multiplied together (weighted equally), and the resulting prior PDF is normalised before being used in Bayes Theorem.

In Fig. 18, we show the computed posterior distributions of the peak energy of the EUV radiation field $E_{\text {peak }}, \log \mathrm{O} / \mathrm{H}+12$, the ionization parameter $\log U$, and the gas pressure $\log P / k$ for the AGN in ShaSS 073, and the HER. The dotted lines show the peak of the 1D marginalized PDF. On each distribution is plotted the model defined by the parameter estimates (brown circle), the peak of the 2D marginalized distribution (blue open triangle) and the projected peak of the full $n$-D PDF (orange open square).

Note that for ShaSS 622, the oxygen abundance falls below the minimum value computed in the theoretical grid, so the derived abundance is only an upper limit. Note also that both $E_{\text {peak }}$ and $\log U$ are appreciably lower in ShaSS 073 than in the HER. These both suggest that the AGN in ShaSS 073 was in a much higher state of activity when the photons now reaching the HER were launched.

The derived parameter estimates with their likely ranges are presented in Table 2 for both the HER and for the two $\mathrm{HII}$ regions. These results confirm that $\log \mathrm{O} / \mathrm{H}+12$ is much lower in ShaSS 622 than in ShaSS 073.

\subsection{Detailed Photoionization Models}

In this section we consider another method of exploring how the physical parameters affect the nebular physics, which involves running tailored photoionization models for the two H II regions and the HER.
This modelling is performed in a similar manner to that described by Dopita et al. (2014). This consists in running a fine grid of models, varying the metallicity and ionization parameter $\log U$ while keeping the input EUV spectrum, gas pressure and dust depletion factors fixed. In order to measure the goodness of fit, we measure the L1-norm, which is the modulus of the mean logarithmic difference in flux (relative to $\mathrm{H} \beta$ ) between the model and the observations;

$$
\mathrm{L} 1=\Sigma_{n}\left|\log \left[\frac{F_{n}(\text { model })}{F_{n}(\text { obs. })}\right]\right| / n .
$$

This weights fainter lines equally with stronger lines, and is therefore more sensitive to the values of the input parameters.

The results of the fit are shown in Table 3. For the two $\mathrm{H}$ II regions the model has an abundance of 0.3 solar $(12+\log (\mathrm{O} / \mathrm{H})=8.2)$, a pressure $\log (P / k)=6.5 \mathrm{~cm}^{-3} \mathrm{~K}$ and ionization parameter $\log U=-2.75$. Together with the diagnostic diagrams, the Bayesian fitting and this detailed models we can be confident that in ShaSS 622 $8.2 \leqslant 12+\log (\mathrm{O} / \mathrm{H}) \leqslant 8.5$.

In the case of the AGN fitting the hardness of the EUV radiation field field is strongly constrained by the [He II] $\lambda 4686$ to [He I] $\lambda 4471$ ratio and the [He I] $\lambda 5876$ to $\mathrm{H} \beta$ ratio, since these are largely determined by the relative number of photons able to ionize $\mathrm{H}^{0}$ to $\mathrm{H}^{+}, \mathrm{He}^{0}$ to $\mathrm{He}^{+}$and $\mathrm{He}^{+}$to $\mathrm{He}^{++}$. Further constraints on the hardness of the radiation field are provided by the [Ne V] $\lambda 3426$ to [Ne III] $\lambda 3868$ ratio and the [Ar IV] $\lambda 4711$ to [Ar III] $\lambda 7136$ ratio. Our best fit model for the HER has $\log E_{\text {peak }}=-1.25$, a hard photon spectral index of -1.9, an abundance of $0.4 \operatorname{solar}(12+\log (\mathrm{O} / \mathrm{H})=8.32)$, a gas pressure of $\log (P / k)=6.2 \mathrm{~cm}^{-3} \mathrm{~K}$ and an ionization parameter $\log U=-2.2$. The computed L1-norm is 0.11 using 32 emission lines.

A quantity of interest for our analysis is the recombination time-scale for the gas in the HER. As the gas freely recombines, the emitted spectrum shifts rapidly to be dominated by the low-ionization species. [O III] therefore rapidly becomes weaker, and [Ne V] disappears, while both lines are strong in the spectrum of HER (Fig. 16 upper panel). The maximum time this process can take is governed by the Hydrogen recombination time-scale. We assume a Case B recombination coefficient at $10^{4} \mathrm{~K}$ of $\alpha=1.58 \times 10^{-13} \mathrm{~cm}^{3} \mathrm{~s}^{-1}$ (Osterbrock \& Ferland 2006). With the same temperature and the estimated pressure in the HER of $\log (P / k)=6.2 \mathrm{~cm}^{-3} \mathrm{~K}$, we can infer an Hydrogen density of $\sim 70 \mathrm{~cm}^{-3}$ and an electron density of $\sim 80 \mathrm{~cm}^{-3}$ (allowing for He to be ionised). This leads to a recombination time-scale of $\mathrm{H}$ within the HER of $\sim 2500 \mathrm{yr}$. The recombination time for [O III] $\left(\alpha=1.72 \times 10^{-11} \mathrm{~cm}^{3} \mathrm{~s}^{-1}\right)$ is $\sim 23 \mathrm{yr}$. 
Table 3. The spectrum of the HER (Fig. 16) and the spectra of two H II regions shown in Fig. 17 compared with their detailed photoionization fits.

\begin{tabular}{|c|c|c|c|c|c|c|}
\hline \multirow[t]{2}{*}{$\lambda(\AA)$} & \multirow[t]{2}{*}{ Line ID } & \multicolumn{2}{|c|}{ HER } & \multicolumn{3}{|c|}{ H II regions } \\
\hline & & Observed & Model & Н II \#1 & H II \#2 & Model \\
\hline 3346 & {$[\mathrm{Ne} \mathrm{v}]$} & $13.19 \pm 0.30$ & 8.39 & & & \\
\hline 3426 & {$[\mathrm{Ne} \mathrm{v}]$} & $35.50 \pm 0.41$ & 23.50 & & & \\
\hline 3729 & {$[\mathrm{O}$ II] } & & & $252.7 \pm 6.5$ & $201.5 \pm 3.2$ & 355.0 \\
\hline 3738 & [O II] & $240.8 \pm 1.6$ & 160.84 & & & \\
\hline 3869 & {$\left[\begin{array}{ll}\mathrm{Ne} & \mathrm{III}\end{array}\right]$} & $73.07 \pm 1.13$ & 84.67 & $21.8 \pm 3.3$ & $26.0 \pm 1.4$ & 52.9 \\
\hline 3889 & $\mathrm{H} \zeta$ & & & $13.1 \pm 1.8$ & $12.8 \pm 1.7$ & 10.5 \\
\hline 3967 & {$\left[\begin{array}{ll}\mathrm{Ne} & \mathrm{III}\end{array}\right]$} & $24.42 \pm 0.63$ & 25.51 & & & \\
\hline 3970 & $\mathrm{H} \epsilon$ & $14.82 \pm 1.96$ & 15.40 & & & \\
\hline 3889 & $\mathrm{H} \zeta$ & & & $13.1 \pm 1.8$ & $12.8 \pm 1.7$ & 10.5 \\
\hline 4068 & {$[\mathrm{~S}$ II $]$} & $3.1 \pm 0.53$ & 1.80 & & & \\
\hline 4076 & {$[\mathrm{~S}$ II] } & $1.0 \pm 0.50$ & 0.58 & & & \\
\hline 4102 & $\mathrm{H} \delta$ & $24.48 \pm 1.60$ & 25.20 & $29.8 \pm 1.5$ & $23.8 \pm 1.2$ & 25.8 \\
\hline 4340 & $\mathrm{H} \gamma$ & $45.96 \pm 0.11$ & 46.74 & $48.7 \pm 1.9$ & $42.2 \pm 1.6$ & 47.0 \\
\hline 4363 & [O III] & $21.31 \pm 0.17$ & 20.65 & $8.7 \pm 3.4$ & $0.4 \pm 0.4$ & 4.2 \\
\hline 4471 & $\mathrm{HeI}$ & $2.80 \pm 0.17$ & 2.81 & & & \\
\hline 4686 & He II & $26.4 \pm 0.39$ & 33.58 & & & \\
\hline 4711 & {$[\mathrm{Ar} I V]$} & $4.12 \pm 0.13$ & 5.73 & & & \\
\hline 4740 & {$[\mathrm{Ar} I V]$} & $3.45 \pm 0.20$ & 4.34 & & & \\
\hline 4861 & $\mathrm{H} \beta$ & $100 \pm 0.80$ & 100 & $100.0 \pm 2.4$ & $100.0 \pm 2.6$ & 100.0 \\
\hline 4959 & [O III] & $292.3 \pm 0.55$ & 331.0 & $116.4 \pm 2.4$ & $109.2 \pm 2.6$ & 120.2 \\
\hline 5007 & [O III] & $876.3 \pm 0.70$ & 956.7 & $349.1 \pm 4.5$ & $327.5 \pm 5.4$ & 347.4 \\
\hline 5200 & {$[\mathrm{~N} \mathrm{I}]$} & $1.25 \pm 0.60$ & 2.85 & & & \\
\hline 5876 & He I & $7.49 \pm 0.10$ & 7.30 & $8.4 \pm 1.3$ & $14.2 \pm 2.1$ & 11.5 \\
\hline 6087 & {$[\mathrm{Fe}$ VII $]$} & $1.45 \pm 0.26$ & 0.44 & & & \\
\hline 6300 & {$[\mathrm{O} \mathrm{I}]$} & $14.43 \pm 1.07$ & 12.19 & $10.0 \pm 2.0$ & $7.1 \pm 3.9$ & 5.6 \\
\hline 6312 & {$[\mathrm{~S}$ III] } & $2.89 \pm 0.69$ & 8.42 & & & \\
\hline 6364 & {$[\mathrm{O} \mathrm{I}]$} & $4.56 \pm 0.36$ & 3.90 & $6.4 \pm 1.6$ & $3.3 \pm 0.9$ & 1.8 \\
\hline 6548 & {$[\mathrm{~N}$ II] } & $7.26 \pm 0.65$ & 5.11 & $4.1 \pm 2.3$ & $7.4 \pm 3.5$ & 7.1 \\
\hline 6563 & $\mathrm{H} \alpha$ & $285.8 \pm 0.30$ & 293.8 & $286.0 \pm 4.5$ & $286.0 \pm 5.5$ & 285.5 \\
\hline 6583 & {$[\mathrm{~N}$ II $]$} & $21.27 \pm 0.96$ & 15.09 & $12.2 \pm 3.0$ & $22.1 \pm 3.0$ & 20.2 \\
\hline 6678 & $\mathrm{H} \mathrm{I}$ & $2.13 \pm 0.50$ & 2.06 & $4.9 \pm 2.0$ & $7.0 \pm 3.5$ & 3.2 \\
\hline 6716 & {$[\mathrm{~S}$ II $]$} & $39.65 \pm 0.43$ & 26.58 & $33.9 \pm 3.5$ & $48.9 \pm 4.5$ & 23.3 \\
\hline 6731 & {$[\mathrm{~S}$ II] } & $27.81 \pm 0.35$ & 18.84 & $22.9 \pm 2.6$ & $35.2 \pm 3.5$ & 19.7 \\
\hline 7136 & [Ar III] & $8.80 \pm 0.61$ & 10.06 & & & \\
\hline 7319 & {$[\mathrm{O}$ II] } & $3.54 \pm 0.99$ & 2.50 & & & \\
\hline 7329 & [O II] & $2.34 \pm 1.05$ & 2.02 & & & \\
\hline 7751 & [Ar III] & $1.89 \pm 0.15$ & 2.41 & & & \\
\hline
\end{tabular}




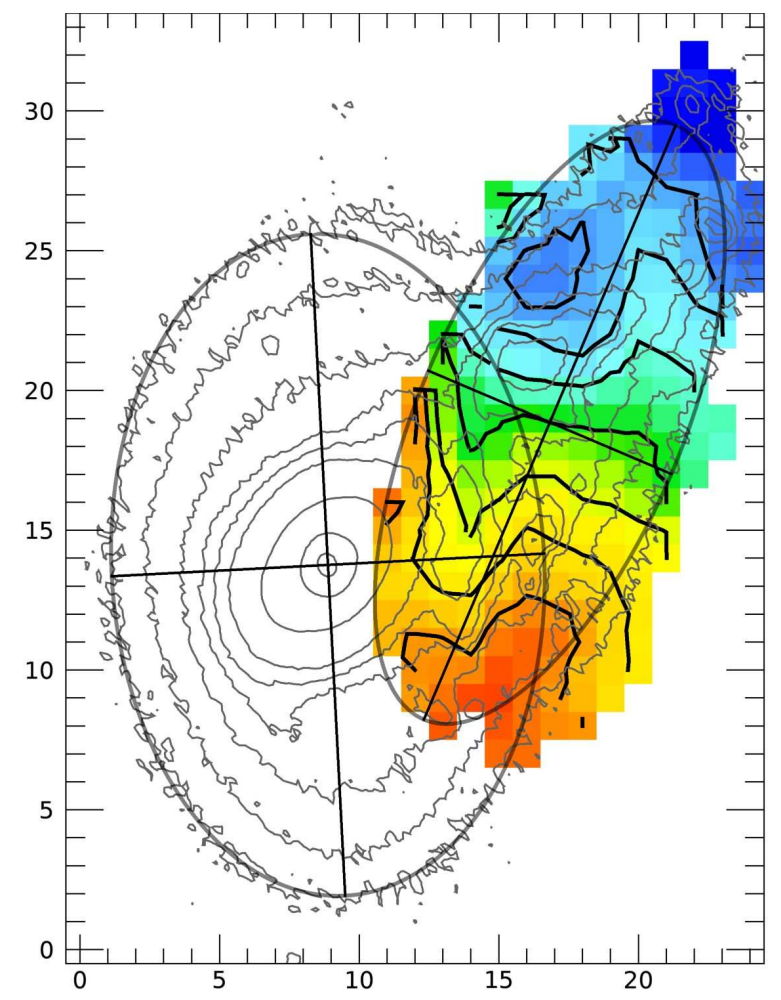

Figure 19. Radial velocity field of the gas of ShaSS 622 . These are the same data shown in Fig. 7, but with a different choice of the kinematic center aimed at revealing the regularity of the velocity field of ShaSS 622 . Ellipses locating the disks of the two galaxies are plotted along with the main axes. Black contours of constant radial velocity are plotted at $25 \mathrm{~km} \mathrm{~s}^{-1}$ intervals, and gray contours are $r$-band isophotes. Scales are in arcseconds in the WiFeS reference frame.

\section{STRUCTURE OF THE SHASS 622-073 SYSTEM}

In this Section we will try to model the threedimensional structure of the galaxy ShaSS 622-073 starting from the observation that the most of the disk of ShaSS 622 is kinematically almost unperturbed. Fig. 19 shows the same radial velocity field of Fig. 7, but assuming a different kinematic center and removing most of the field of ShaSS 073 for clarity. An ellipse was sketched following the external $r$-band isophotes of ShaSS 073 in the area where there is no clear superposition between the two galaxies. Another ellipse encircles the area in which the kinematic field of ShaSS 622 appears to be fairly regular, with the center adjusted to maximize its symmetry. The requirement of maximum symmetry gave little room for the choice of the center, which is confined within about \pm 1 pixel from the chosen position.

The regularity of the velocity field inside the ellipse implies that the motion of the gas is still only weakly perturbed by the interaction, in agreement with the very early stage of interaction claimed for in the preceding

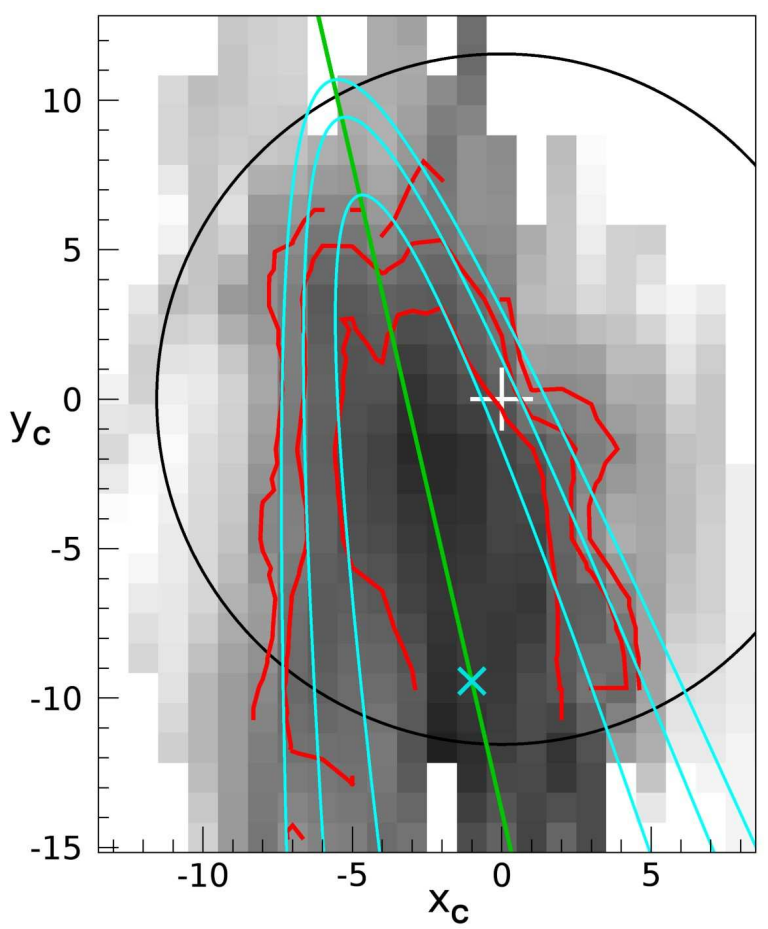

Figure 20. The distribution of $[\mathrm{O}$ III $] / \mathrm{H} \beta$ on the disk of ShaSS 622 (black circle), mapped by gray tones and by the red curves of constant ratios $\log ([\mathrm{O} \mathrm{III}] / \mathrm{H} \beta)=0.85,0.9,0.95$ (outside-in). The green line marks the preferential direction of the distribution of line ratios. The white cross is the center of ShaSS 622 and the cyan cross is the assumed intersection of the ionization cone axis with the disk. The cyan conics are the intersections with the disk of cones of semi-aperture of $7^{\circ}, 10^{\circ}, 12^{\circ}$ (inside-out). The outermost conics delimits the HER. The units on the axes are arcsecs (measured from the center of ShaSS 622).

Sections $^{9}$. As a consequence of this, we can safely assume that the gas within the sketched ellipse still lies in the galactic plane. As we will show in the following, this has a significant impact on our knowledge of the ShaSS 622-073 system, because this gas constitutes a nearly flat 'projection screen' being hit by the ionization cone produced by the AGN.

Our analysis starts from the properties of the disks (axis ratios and position angles) and the distribution of the $[\mathrm{O}$ III $] / \mathrm{H} \beta$ ratio on the disk of ShaSS 622 . The derivations are presented in Appendix A. The axis ratio of ShaSS $622, b / a \sim 0.41$, corresponds to an inclination (disk to sky plane) of $\pm 66^{\circ}$ (assuming an infinitely thin layer of gas, appropriate for a late-type spiral). For the inclination we adopt the notation $k_{6} \cdot i_{6}= \pm 66^{\circ}$, where $i_{6}=66^{\circ}$ and the parameter $k_{6}= \pm 1$ determines which side of the disk of ShaSS 622 is facing the observer and

\footnotetext{
9 The lack of axial symmetry, apparent from the twisting of iso-velocity contours, might be either the initial effect of the interaction or the effect of a pre-existing bar causing non-circular motions
} 
in turn the orientation of the observer with respect to the whole system (see below).

Let us now introduce a reference system $\mathcal{C}:\left(x_{\mathcal{C}}, y_{\mathcal{C}}, z_{\mathcal{C}}\right)$ (see Appendix A), with the origin and the $z_{\mathcal{C}}$ axis coincident with the center and the rotation axis of ShaSS 662 respectively. The transformation to this system is achieved by a rotation around the apparent major axis of the galaxy by one of the two angles $k_{6} i_{6}= \pm 66^{\circ}$. In the Appendix $\mathrm{A}$ it is shown that this transformation of coordinates can't be applied a priori to ShaSS 073 unless we have an independent constraint on its position.

We will now show that the shape of the illuminated area on the disk of ShaSS 622 , as defined by the $[\mathrm{O} \mathrm{III}] / \mathrm{H} \beta$ ratio, allows us to determine the direction to the AGN in the reference system $\mathcal{C}$, thus providing the needed constraint.

We simulate the transformation to system $\mathcal{C}$ of the disk of ShaSS 622 by expanding the observed map of $[\mathrm{O} \mathrm{III}] / \mathrm{H} \beta$ by a factor $1 / \cos \left(i_{6}\right)$ in the direction orthogonal to the apparent major axis. This stretch factor transforms the disk of ShaSS 622 into a circle and can be shown to correspond exactly to the rotation of $i_{6}$ degrees for a thin disk ${ }^{10}$.

Fig. 20 shows the distribution of $[\mathrm{OIII}] / \mathrm{H} \beta$ on the disk of ShaSS 622, mapped by gray tones and by the red curves of constant $\log ([\mathrm{O} \mathrm{III}] / \mathrm{H} \beta)=0.85,0.9,0.95$ from outside-in (to compare with the values in Fig. 13). The map and the curves clearly indicate a preferential direction, marked with a green line, which should correspond to the projection on the galaxy plane of the axis of the ionization cone, and therefore also marks the direction to the AGN. As it is shown in the Appendix A, this is the constraint on the position of ShaSS 073 which allows us to derive a 3-D model for the two galaxies.

Knowing now the relative positions of the two galaxies and that the intersection of the cone axis with the plane of ShaSS 622 must lie along the green line in Fig. 20, we can determine, for any semi-aperture $\psi$ of the cone, the shape of the area illuminated by the radiation from the AGN and compare this with the observed curves of constant $[\mathrm{O} \mathrm{III}] / \mathrm{H}_{\beta}$. It turns out that the range of positions and cone semi-apertures leading to the observed shape of the illuminated area is relatively small. In other words, the observed distribution of $[\mathrm{O} \mathrm{III}] / \mathrm{H} \beta$ imposes tight constraints on the geometry (aperture and orientation) of the ionization cone. The assumed intersection of the cone axis with the plane of ShaSS 622 is marked by a cyan cross in Fig. 20, while the intersections of cones

${ }^{10}$ We performed numerical simulations with different shapes and spatial sampling to assess the ability to recognize in this way the original shapes on a plane from a skewed line of sight. We found that this method recovers shapes quite accurately, except for extremely poor spatial sampling, which is not our case.

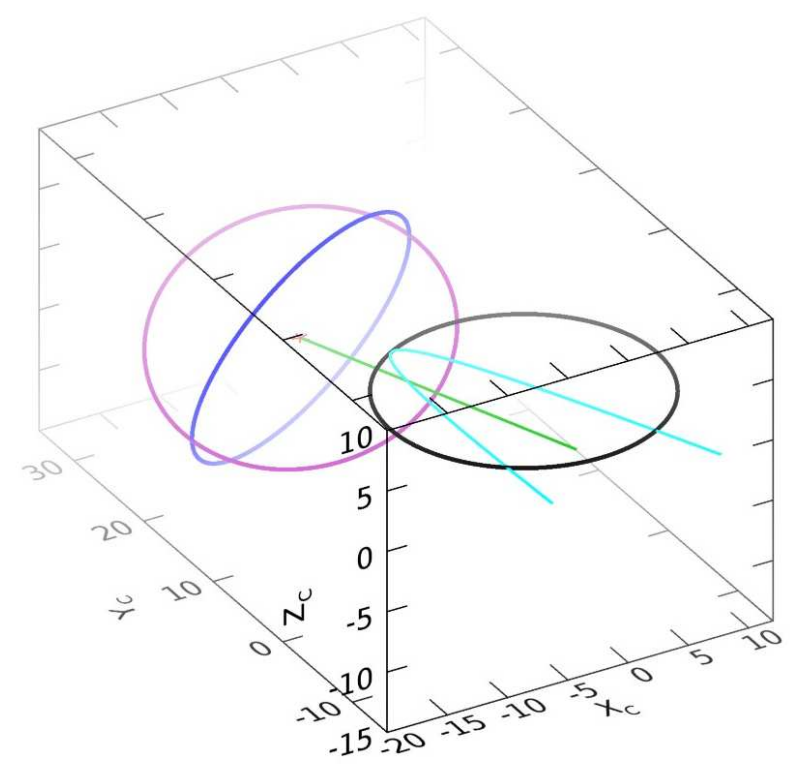

Figure 21. View of the ShaSS 073-622 pair in reference system $\mathcal{C}$, where the axis of ShaSS 662 coincides with $z_{\mathcal{C}}$ (see Appendix A). The disks of ShaSS 622 (black) and ShaSS 073 (blue and purple for $k_{7}= \pm 1$ respectively) are shown. Also shown are the axis of the ionization cone (green line) and the intersection of the cone of semi-aperture $12^{\circ}$ with the disk of ShaSS 622 (cyan curve, delimiting the HER). The units on the axes are kpc.

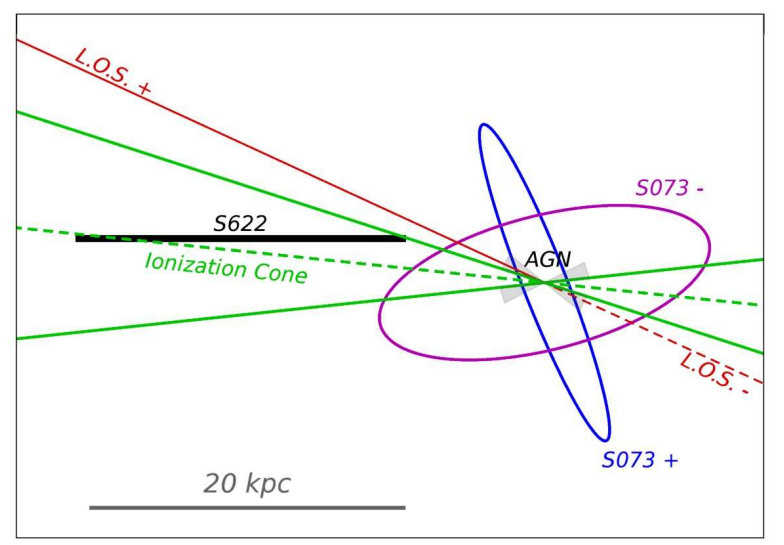

Figure 22. View of the ShaSS 073-622 system with the axis of the ionization cone (green dashed line) in the plane of the plot. The disks of ShaSS 622 and ShaSS 073 (in the two possible orientations according to the sign of $k_{7}$ ) are shown, with ShaSS 622 viewed edge-on. The two continuous green lines are the walls of the ionization cone, assumed to have a semi-aperture $\psi=12^{\circ}$. The two possible line-of-sights are shown as a continuous $\left(k_{6}=+1\right)$ and dashed $\left(k_{6}=\right.$ $-1)$ red lines. The gray area sketches the angular extent of the outflow which roughly reproduces the geometry of the observations. 
Table 4. Parameters defining the spatial structure of the ShaSS 622-073 system. The intervals of parameters which are broadly consistent with the data are indicated in square brackets (see Appendix A.

\begin{tabular}{lcc}
\hline \hline & $k_{7}=-1$ & $k_{7}=+1$ \\
\hline distance between the centers of the galaxies & \multicolumn{2}{c}{$21 \mathrm{kpc}[19-24]$} \\
\hline distance along the line of sight & $19 \mathrm{kpc}[17-22]$ \\
\hline angle between their disks & $27^{\circ}[22-32]$ & $69^{\circ}[63-75]$ \\
\hline semi-aperture of ionization cone & \multicolumn{2}{c}{$12^{\circ}[12-15]$} \\
\hline angle between ShaSS 073 axis and cone axis & $71^{\circ}[66-78]$ & $28^{\circ}[21-34]$ \\
\hline angle between cone axis and line of sight & $22^{\circ}[20-25]$ \\
\hline angle between the cone axis and the disk of ShaSS 622 & $6^{\circ}[2-10]$ \\
\hline
\end{tabular}

with semi-apertures of $7^{\circ}, 10^{\circ}$, and $12^{\circ}$ are shown by the cyan conics. We remark here that our model gives a purely geometric prediction in terms of cone-plane intersection, while the actual distribution of $[\mathrm{O}$ III $] / \mathrm{H} \beta$ depends on much more complex factors, like, for instance, the distribution of the nuclear radiation within the cone, the spatial distribution of dust in the disk and the local physical conditions of the gas. Taking into account the above caveats, these curves overlap fairly well with those of constant $[\mathrm{O} \mathrm{III}] / \mathrm{H} \beta$, and we adopt $\psi=12^{\circ}$ as the semi-aperture of the ionization cone of the AGN.

The overall picture suggested by our model is depicted in Figs. 21 and 22 and summarized in Table 4. Fig. 21 shows the galaxies embedded in the reference system $\mathcal{C}$ from an arbitrary angle of view. The disk of ShaSS 622 (black) is affected by the ionization cone within the area limited by the cyan curve (the HER). The axis of the ionization cone is plotted as a green line running from the AGN to the disk of ShaSS 622. The two possible orientations of the disk of ShaSS 073, which are identified by the parameter $k_{7}$, are shown in blue $\left(k_{7}=+1\right)$ and purple $\left(k_{7}=-1\right)$. Fig. 22 offers a view perpendicular to the ionization cone axis (dashed green line). The surface of the ionization cone is represented by the continuous green lines, and the galaxies discs are represented as in Fig. 21, with ShaSS 622 viewed edge-on. The red lines mark the two different line of sights according to the sign of $k_{6}$. Note that for $k_{6}=+1$ ShaSS 622 is closer to the observer than ShaSS 073 and vice-versa. The gray areas schematically indicate the corona of the cone where the outflow is taking place.

The distance between the centers of the two galaxies turns out to be $21 \mathrm{kpc}$, with their disks being inclined by $27^{\circ}\left(69^{\circ}\right)$ for $k_{7}=+1(-1)$ with respect to each other. The axis of the ionization cone is inclined by $22^{\circ}$ with respect to the line of sight, by $71^{\circ}\left(28^{\circ}\right)$ for $k_{7}=+1(-1)$ with respect to the rotation axis of the host galaxy, and $6^{\circ}$ with respect to the disk of ShaSS 622 . We notice however that the angle between the cone axis and the disk of
ShaSS 073 for $k_{7}=-1$ is just $19^{\circ}$, while we observe the outflow extending beyond $22^{\circ}$ from the cone axis (see Fig. 22 and sixth row of Table 4). This would imply that the outflow impacts the disk directly, giving rise to strong kinematical perturbations. These perturbations should be observable in the gas kinematics on both sides of the AGN, since the outflow would be tangential with the disk. Since no such perturbation is seen, we tend to conclude that the angle between the ionization cone and the axis (disk) of the AGN host galaxy is $28^{\circ}\left(62^{\circ}\right)$, corresponding to $k_{7}=+1$, which also implies that the angle between the two disks is $69^{\circ}$ (blue models in Figs. 21 and 22).

The kinematics of the gas also allows us to constrain the orientation of the system with respect to the line of sight. In preceding Sections (Sects. 5.2 and 6.2) we discussed the area of the northern disk of ShaSS 073 in which the gas is characterised by a second kinematical component, which is blue-shifted with respect to the disk and with somewhat higher velocity dispersion, but which does not belong to the outflow. The natural explanation for this kinematics is the tidal interaction with ShaSS 622, which is starting to pull the gas out of the disk of ShaSS 073 (blue-shift) inducing at the same time turbulence (high $\sigma$ ). Looking at Fig. 22, this can be the case if and only if we are observing the system from the 'upper left' $\left(k_{6}=+1\right)$, otherwise the perturbed gas would be red-shifted. We therefore tentatively ascribe the second kinematical component of the gas to the onset of the tidal perturbation in the disk of ShaSS 073 . In addition, being this component blue-shifted, we infer that the system is oriented as parametrized by $k_{6}=+1$ (Fig. 22), with ShaSS 622 closer to us (by $\sim 19 \mathrm{kpc}$ ) than the AGN-host galaxy.

To assess the reliability of this picture, we changed, within reasonable limits, the initial assumptions concerning the position and orientation of the two disks and derived from these new values the parameters of the 3-D structure. The results, given in square brackets in Ta- 
ble 4, represent extreme cases which are still marginally consistent with the data. This process is detailed in the Appendix A. The values in Table 4 show that our description of the spatial structure of the ShaSS 073-622 system is robust against reasonable changes of the initial assumptions.

\section{DISCUSSION}

\subsection{Allocating the $A G N$ radiation power}

An interesting question is whether the flux from the AGN is consistent with the outflow being powered by radiation pressure and, more important, can ionize the gas in ShaSS 622 at the observed level.

An upper limit to the power needed to maintain the outflow may be obtained starting from its high ionization parameter $(\log U \sim-1.0$, see Sect. 7). Such a high value indicates that the gas pressure is determined by the radiation pressure in this component. Furthermore, for this component, the $[\mathrm{S} \mathrm{II}] \lambda \lambda 6731 / 6717$ ratio is $\sim 1.3$. Taking into account the errors, this indicates an electron density $2.8 \leqslant n_{e} \leqslant 3.5 \mathrm{~cm}^{-3}$. When the gas is radiation-pressure dominated, we can set the gas pressure $P_{g a s}=L / 4 \pi R^{2} c$, where $L$ is the luminosity of the source, and $R$ is the distance of the gas parcel from the source. For ShaSS 073, the emitting region is unresolved within the central spaxel, so we can only compute an upper limit on the luminosity, assuming that the emitting region fills the spaxel. This implies $R \sim 500 \mathrm{pc}$ and hence $\log L \lesssim 45.0 \mathrm{erg} \mathrm{s}^{-1}$, which is actually larger than the bolometric luminosity of the AGN $\log L=44.39 \mathrm{erg} \mathrm{s}^{-1}$ derived in Sec. 2.3.

The [O III] luminosity of the gas in the HER can be used to estimate the AGN luminosity. The integrated [O III] $\lambda 5007$ flux in this region is $2.25 \times 10^{42} \mathrm{erg} \mathrm{s}^{-1}$. Using the geometric model of Sec. 8, we estimate that the ionized region intercepts a solid angle $\Omega=0.0686 \mathrm{sr}$ as seen by the AGN in the main galaxy. It follows that the total omnidirectional [O III] flux would be $4.12 \times 10^{44} \mathrm{erg} \mathrm{s}^{-1}$. In the photoionization model presented above, the [O III] line represents $8.87 \%$ of the bolometric EUV flux. Therefore, we can estimate the luminosity of the AGN as $\log L=45.66 \mathrm{erg} \mathrm{s}^{-1}$.

Combining our photoionization model estimate with the bolometric luminosity given by the SED fit, $\log L=$ $44.39 \mathrm{erg} \mathrm{s}^{-1}$, we conclude that the AGN radiation required to excite the gas inside the HER is $\sim 20$ times the current luminosity of the AGN. This is also supported by the Bayesian analysis which obtains lower values of both $E_{\text {peak }}$ and $\log U$ for the AGN than in the HER. Taking into account the maximum semi-aperture of the cone allowed by the data $\left(15^{\circ}\right.$, see Appendix A), we obtain an upper limit for the solid angle of $\Omega=0.1070$, which would reduce the above factor from 20 to 13 .
Considering the relative positions of the AGN and ShaSS 622 (Fig.22) and the fact that the ionized gas recombination times are very short compared to the light travel time (Sect. 7.3), we infer that the AGN luminosity is dropped by a factor 20 within the last $\sim 3 \times 10^{4} \mathrm{yr}^{11}$.

The bolometric luminosity of the AGN has been estimated in Sect. 2.3 from the emission at 5-6 $\mu \mathrm{m}$ which comes from the optically-thick dust torus surrounding the central engine and accretion disk. It is therefore interesting to compare the above time-scale with the time needed to turn this mid-infrared emission off. The temperature of the dust and resulting infrared emission responds rapidly to changes in the accretion rate, as confirmed by quasars/AGN being variable in the midinfrared on 1-10 year time-scales (Kozłowski et al. 2016), and the tight correlation between nuclear mid-infrared and X-ray luminosities of AGNs (e.g. Lutz et al. 2004; Mateos et al. 2015). In the case of an AGN being instantaneously quenched, the dust torus should cool from $1000 \mathrm{~K}$ to several tens of Kelvin and the $5-6 \mu \mathrm{m}$ emission drops by a factor $\sim 300$ within $\sim 30 \mathrm{yr}$ (Ichikawa \& Tazaki 2017).

\subsection{Relation of ShaSS 073-622 with light echoes}

Assuming that our picture of the spatial structure of the system is correct, and also knowing the relative velocity of the galaxies along the line of sight $\left(130 \mathrm{~km} \mathrm{~s}^{-1}\right)$, there is still one major unknown preventing us to envisage the future of our system - the spatial relative velocity.

Depending on this velocity, the interaction could result from a complete merging of the two galaxies to just a pass-by, with the gas probably being stripped off ShaSS 622 and captured by the AGN host. In any case, it is likely that the gas of ShaSS 622 will cross the ionization cone of the AGN, while in the meantime it is likewise possible that the AGN activity will change in intensity.

The ShaSS 073-622 system can be related to the green bean (GB) sample of Schirmer et al. (2013) at redshifts $z=0.2-0.6$ characterized by strong [O III] fluxes. Likewise this sample, the ShaSS 073-622 system is characterized by the presence of an AGN and an EELR powered by it and the GBs are Seyfert-2 galaxies as ShaSS 073.

The luminosities at $24 \mu \mathrm{m}$ and in the [O III] $\lambda 5007$ line of our system are also consistent with the sample of 'quasar light-echo' candidates of Schirmer et al. (2013). We remark however that the fact that the AGN flux is currently in a faded phase is to be considered incidental, because this variation time scale is much shorter (by a

\footnotetext{
11 This value refers to the gas in the HER closest to the AGN, which will be the first affected by the fading of the AGN.
} 
factor $\sim 10^{3-4}$ ) than the interaction/merging time scale.

Another feature in common with most of light echo objects is the fact that our AGN is radio-weak. This also holds for several objects of the Schirmer et al.'s sample as well as for Hanny's Voorwerp (HV, Rampadarath et al. 2010), which differently from the GBs consists of an ionized nebula detached from the galaxy IC 2497 containing the powering AGN. Keel et al. (2012a) assembled a sample HV-like galaxies from the Galaxy Zoo Survey (hereafter GZ sample, see also Sect. 1) which, as ShaSS 073-622, are lower in redshift than the GBs. The diagnostic diagrams in Fig. 13 can be compared to the line ratios of the GZ sample (see Fig. 4 of Keel et al. 2012a) and the line ratios of the GB SDSS J224024.1092748 which has been studied in detail by Davies et al. (2015b). The gas in the HER populates the diagrams where line ratios typical of Seyfert galaxies are expected in agreement with the finding of the other works. In particular, the value of $\log ([\mathrm{O} \mathrm{III}] / \mathrm{H} \beta) \sim 1$ is completely consistent with those measured for the mentioned samples.

Previous studies invoke the role of the interaction to explain their observations. In the GB SDSS J224024.1092748 (Davies et al. 2015b), the EELR is interpreted as the remnant of a quasar-driven outflow triggered by a preceding galaxy interaction. Most (73\%) of the objects in the GZ sample are classified as interacting, merging or post-merging systems, and Keel et al. (2015) identified in eight GZ galaxies tidal tails, shells and chaotic dust structures and systematically lower metallicity of the ionized gas with respect to the nuclei. This supports the hypothesis that the the EELRs around local AGNs are illuminated tidal debris and ShaSS 073-622 seems to be the likely progenitor of a GB- or HV-like object.

\section{SUMMARY AND CONCLUSIONS}

In the framework of our project aimed at the study of galaxies in the process of being transformed in the Shapley supercluster by using the multi-band ShaSS data-set (Merluzzi et al. 2015), we targeted a system consisting in a galaxy hosting a Seyfert-2 nucleus (ShaSS 073) and another galaxy (ShaSS 622), which are in the initial phase of interaction. This study is based on integral-field optical spectroscopy as well as on photometry from the FUV to the IR and radio.

The AGN-host galaxy is the more massive of the pair $\left(\mathrm{M}^{*}=5.7 \times 10^{10} \mathrm{M}_{\odot}\right)$ and is probably of morphological type (R)SB0a, while the mass of the companion (Sbc) is a factor $\sim 10$ lower. The available fluxes from FUV (GALEX) through optical and IR (IRAS, Spitzer) allow to recover the spectral energy distribution of the nucleus of ShaSS 073, which shows both the contribution of the AGN and star formation. The radio luminosity of $\mathrm{L}_{1.4 G H z} \sim 2 \times 10^{22} \mathrm{~W} \mathrm{~Hz}^{-1}$ most probably comes from star formation at a rate of $\mathrm{SFR} \sim 6 \mathrm{M}_{\odot} \mathrm{yr}^{-1}$. Both the SED and the NIR-MIR fluxes give for the AGN a bolometric luminosity of $2.4 \times 10^{44} \mathrm{erg} \mathrm{s}^{-1}$.

The chemical abundances of the gas phase are significantly different in the two galaxies, being $\sim 1.5$ supersolar in the AGN host galaxy and $\sim 0.4$ solar in the companion. The dust content in the two galaxies also reflects this difference. This marked difference is crucial in recognising the gas from the two galaxies all over the system.

Clear signs of interaction between the two galaxies are i) the perturbation of the kinematics of the portion of the disk of ShaSS 622 closest to ShaSS 073; ii) gas from the disk of ShaSS 073 is pulled toward the companion and iii) the distortion of the external ring of ShaSS 073. However, the perturbation is still weak in the sense that most of the original rotational motion of the two disks is in place and the two gas populations appear to be still well separated. Moreover, the estimated distance of $21 \mathrm{kpc}$ between the two galaxy centers is consistent with this being their initial approach.

The most striking feature of the ShaSS 073-622 system is the high excitation of the gas over a large area $\left(\sim 170 \mathrm{kpc}^{2}\right)$ of the disk of ShaSS 622. The gas is excited by the AGN, as is demonstrated by different kinds of line-ratio diagnostics. The rest of the gas in ShaSS 622 consists of normal Hil regions. Around the center of ShaSS 073, high-velocity $\left(v_{R} \sim 440 \mathrm{~km} \mathrm{~s}^{-1}\right.$ $\sigma \sim 490 \mathrm{~km} \mathrm{~s}^{-1}$ ) gas reveals the presence of an AGN outflow extending out to $\sim 2-3 \mathrm{kpc}$ and ejecting the dust from the nuclear region, as shown by the high values of the attenuation around the galaxy center. The excitation of the external gas and the outflow demonstrate the presence of the hollow bi-cone structure common in (type 2) AGNs.

The analysis of emission-line fluxes in the different areas of the system reveals, in addition to the differences in metallicity, the different status of ionization in i) the AGN (log $U \sim-3)$; ii) in the HER $(\log U \sim-2.4)$; iii) the $\mathrm{H}$ II regions $(\log U \sim-3)$. The value of the ionization parameter of the AGN is lower than in the HER, and the same is true for the peak energy of the radiation. This is contrary to the expectations, considering that the HER should be powered by the AGN, and this is further motivation to investigate the radiation energy balance of the system.

The power needed to excite the gas in the HER is $4.6 \times 10^{45} \mathrm{erg} \mathrm{s}^{-1}$, which is a factor 20 larger than the AGN bolometric luminosity.

The natural explanation of this discrepancy is that the AGN flux has faded in the time the radiation needs to travel from the nucleus of ShaSS 073 to the disk of ShaSS 622 , which is $\sim 3 \times 10^{4} \mathrm{yr}$, which we therefore assume as an upper limit of the variability time scale ex- 
perienced by the AGN. Similar time-scales are obtained by Keel et al. (2015) $\left(\sim 5 \times 10^{4} \mathrm{yr}\right)$ and Keel et al. (2017) $\left(\sim 2 \times 10^{4} \mathrm{yr}\right)$.

It is of course impossible to predict whether the ShaSS 073-622 system will turn into the kind of objects belonging to the family of GBs or HV-like objects, but it appears that all ingredients are already in place. Because of these features, the ShaSS 073-622 system could well constitute the first example of a parent of $\mathrm{HV}$ like objects. Considering the fact that both the GB and the HV-like objects are rare, our discovery suggests that either we caught an almost unique event or such phenomenon has a short time-scale and is not intrinsically 'dramatic'. Since we do not trust the fortune so much, we tend to believe that similar events are less rare than previously believed and we expect that current (e.g. MANGA) and future surveys with IFS will deliver other such systems improving the knowledge of this complex phenomenon.

We thank the anonymous referee for her/his comments which helped to improve this work. This work is based on data collected with (i) $\mathrm{WiFeS}$ at the $2.3 \mathrm{~m}$ telescope of the Australian National University at Siding Spring (Australia) and (ii) OmegaCAM at the ESO INAFVLT Survey Telescope and VIRCAM at VISTA, both at the European Southern Observatory, Chile (ESO Programmes 088.A-4008, 090.A-0094, 090.B-0414, 093.A0465. The optical imaging is collected at the ESO VLT Survey Telescope using the Italian INAF Guaranteed Time of Observations. This publication makes use of data products from i) WISE, which is a joint project of the University of California, Los Angeles, and the Jet Propulsion Laboratory/California Institute of Technology, funded by the National Aeronautics and Space Administration and ii) GALEX (Galaxy Evolution Explorer) is a NASA Small Explorer launched in April 2003. We gratefully acknowledge NASAs support for construction, operation and science analysis for the GALEX mission, developed in cooperation with the Center National dEtudes Spatiales of France and the Korean Ministry of Science and Technology. P. Merluzzi and G. Busarello acknowledge financial support from PRIN-INAF2014: Galaxy Evolution from Cluster Cores to Filaments (PI B.M. Poggianti). M. A. Dopita acknowledges the support of the Australian Research Council (ARC) through Discovery project DP16010363. This research is supported by an Australian Government Research Training Program (RTP) Scholarship.

Software: PyWiFeS (Childress et al. 2014), LZIFU (Ho et al. 2016), Mappings 5.0 (Sutherland et al. 2017, in prep.), Starburst 99 (Leitherer et al. 1999), IZI (Blanc et al. 2015), NebulaBayes (Thomas et al. in prep.)

\section{APPENDIX}

\section{A. GEOMETRY OF THE SYSTEM}

The transformation from the reference system of the WiFeS frame (system $\mathcal{A}$ ) to system $\mathcal{B}$, where ShaSS 622 is centered at the origin, with the major axis along $x_{\mathcal{B}}$ is given by:

$$
\begin{aligned}
& x_{\mathcal{B}}=\left(x_{\mathcal{A}}-x_{\mathcal{A}, 6}\right) \cdot \cos (\alpha)+\left(y_{\mathcal{A}}-y_{\mathcal{A}, 6}\right) \cdot \sin (\alpha) \\
& y_{\mathcal{B}}=-\left(x_{\mathcal{A}}-x_{\mathcal{A}, 6}\right) \cdot \sin (\alpha)+\left(y_{\mathcal{A}}-y_{\mathcal{A}, 6}\right) \cdot \cos (\alpha) \\
& z_{\mathcal{B}}=z_{\mathcal{A}}
\end{aligned}
$$

where $\left(x_{\mathcal{A}, 6}, y_{\mathcal{A}, 6}\right)$ are the coordinates of the center of ShaSS 622 in system $\mathcal{A}$ and $\alpha=67^{\circ}$ is the angle between the major axis of the galaxy and $\mathbf{x}_{\mathcal{A}}$. The coordinate axis $z_{\mathcal{B}}$ corresponds to the line of sight.

A subsequent transformation to system $\mathcal{C}$ brings the disk of ShaSS 622 on the coordinate plane $z_{\mathcal{C}}=0$ :

$$
\begin{aligned}
& x_{\mathcal{C}}=x_{\mathcal{B}} \\
& y_{\mathcal{C}}=y_{\mathcal{B}} \cdot \cos \left(k_{6} i_{6}\right)-z_{\mathcal{B}} \cdot \sin \left(k_{6} i_{6}\right) \\
& z_{\mathcal{C}}=y_{\mathcal{B}} \cdot \sin \left(k_{6} i_{6}\right)+z_{\mathcal{B}} \cdot \cos \left(k_{6} i_{6}\right)
\end{aligned}
$$

where $k_{6} i_{6}= \pm 66^{\circ}$. In these coordinates the rotation axis of ShaSS 622 is along $z_{\mathcal{C}}$. We set $k_{6}=1$ when the farther side of the disk is on the East in the sky.

Let us denote with the subscript $A$ (for 'AGN') the position of the center of ShaSS 073 and rewrite the above equations as

$$
\begin{aligned}
x_{\mathcal{C}, A} & =x_{\mathcal{B}, A} \\
y_{\mathcal{C}, A} & =y_{\mathcal{B}, A} \cdot \cos \left(k_{6} i_{6}\right)-z_{\mathcal{B}, A} \cdot \sin \left(k_{6} i_{6}\right) \\
z_{\mathcal{C}, A} & =y_{\mathcal{B}, A} \cdot \sin \left(k_{6} i_{6}\right)+z_{\mathcal{B}, A} \cdot \cos \left(k_{6} i_{6}\right)
\end{aligned}
$$


This is a system of three equations with six variables of which only two are known: $x_{\mathcal{B}, A}$ and $y_{\mathcal{B}, A}$. To solve the system for the coordinates of the AGN in system $\mathcal{C}$ we need another constraint between the variables. Such a constraint is the projection of the axis of the ionization cone in the plane $z_{\mathcal{C}}=0$, which relates the coordinates of the AGN in that plane. The equation of this projection is

$$
y_{\mathcal{C}}=P x_{\mathcal{C}}+Q
$$

with $P$ and $Q$ determined from the green line in Fig. 20. This equation reduces to three the number of unknown variables in the above system A3, making it possible to derive the full spatial position of the AGN. For instance, eqs. A3 and A4 lead to:

$$
z_{\mathcal{B}, A}=\frac{y_{\mathcal{B}, A} \cdot \cos \left(k_{6} i_{6}\right)-P \cdot x_{\mathcal{B}, A}-Q}{\sin \left(k_{6} i_{6}\right)},
$$

and eventually to the position of the AGN in system $\mathcal{C}$.

It is now possible to determine the distance $D$ between the centers of ShaSS 622 and ShaSS 073

$$
D=\left(x_{\mathcal{C}, A}^{2}+y_{\mathcal{C}, A}^{2}+z_{\mathcal{C}, A}^{2}\right)^{\frac{1}{2}}
$$

since the center of ShaSS 622 is in the origin.

To derive the relative orientation of the two disks we compute the angle between the axis of ShaSS 073 and $z_{\mathcal{C}}$, which is the axis of ShaSS 622. The direction vector of the axis of ShaSS 073 in system $\mathcal{B}$ is given by

$$
\mathbf{G}=\sin \left(k_{7} i_{7}\right) \cdot \sin (\phi) \mathbf{x}_{\mathcal{B}}-\sin \left(k_{7} i_{7}\right) \cdot \cos (\phi) \mathbf{y}_{\mathcal{B}}+\cos \left(k_{7} i_{7}\right) \mathbf{z}_{\mathcal{B}}
$$

where $i_{7}=50^{\circ}$ is the inclination derived from the axis ratio and assuming a disk of finite thickness 0.13 appropriate for an early-type disk. We introduce the parameter $k_{7}= \pm 1$, with $k_{7}=+1$ corresponding to the case where the eastern side disk in the sky is nearer to the observer. The angle $\phi$ is the angle of the major axis with $x_{\mathcal{B}}$, and $\left(\mathbf{x}_{\mathcal{B}}, \mathbf{y}_{\mathcal{B}}, \mathbf{z}_{\mathcal{B}}\right)$ are the direction vectors of system $\mathcal{B}$.

The angle $\gamma$ between $\mathbf{G}$ and the axis $z_{\mathcal{C}}$ is given by the scalar product $\cos (\gamma)=\mathbf{G} \cdot \mathbf{z}_{\mathcal{C}}$, which gives

$$
\cos (\gamma)=\cos \left(k_{7} i_{7}\right) \cdot \cos \left(k_{6} i_{6}\right)-\sin \left(k_{7} i_{7}\right) \cdot \cos (\phi) \cdot \sin \left(k_{6} i_{6}\right)
$$

For the study of the geometry of the AGN ionization cone and the interaction with the disc of the companion galaxy, let us start considering a circular cone of semi-aperture $\psi$ with its axis along $w$ in a reference system $(u, v, w)$, and with the vertex at $(0,0,-h)$. The equation of this cone is

$$
u^{2}+v^{2}=\tan (\psi)^{2} \cdot(h+w)^{2} .
$$

The intersection of the cone with the plane $w=0$ is a circle of radius $h \cdot \tan (\psi)$. Let us consider another reference system $(x, y, z)$ with the same origin, and the $y$ axis coincident with the $v$ axis, but rotated counter-clockwise by an angle $\xi$ around $v$. In this system the equation of the cone becomes

$$
(x \cdot \cos (\xi)-z \cdot \sin (\xi))^{2}+y^{2}=\operatorname{tg}(\psi)^{2} \cdot(x \cdot \sin (\xi)+z \cdot \cos (\xi)+h) .
$$

This is the equation of a cone of semi-aperture $\psi$ whose axis lies on the $y=0$ plane and is inclined by an angle $\xi$ (positive if measured clockwise) with respect to the $z$ axis. The vertex of the cone lies in the $z<0$ semi-space at a distance $h$ from the origin. The intersection of the cone with the plane $z=0$ becomes

$$
D \cdot x^{2}-2 \cdot \tan (\psi)^{2} \cdot h \cdot \sin (\xi) \cdot x+y^{2}=\tan (\psi)^{2} \cdot h^{2}
$$

where

$$
D=\cos (\xi)^{2}-\tan (\psi)^{2} \cdot \sin (\xi)^{2}
$$

The sign of $D$ determines which type of conics is the intersection of the cone with the plane. Since by construction both $\psi$ and $\xi$ belong to the interval $\left[0, \frac{\pi}{2}\right]$, the conditions $D \geq 0$ implies $\frac{\pi}{2} \geq \psi+\xi$. Note that $D=0$, i.e. $\psi+\xi=\pi / 2$, implies that the $x$-axis is parallel to the surface of the cone, so that the intersection is the parabola

$$
x=\frac{y^{2}}{2 \cdot \tan (\psi)^{2} \cdot h \cdot \sin (\xi)}-\frac{h}{2 \cdot \sin (\xi)} .
$$

If $D \neq 0$, after some algebra, we obtain

$$
\frac{\left(x-x_{o}\right)^{2}}{A^{2}}+\operatorname{sgn}(D) \cdot \frac{y^{2}}{B^{2}}=1
$$


Table A1. Ranges of the initial parameters adopted in order to estimate the reliability of the model. Values within the ranges generate the ellipses shown in Fig. A1. The subscript ' 0 ' indicates the quantities of the model adopted in Sec. 8. Units are kiloparsecs and degrees.

\begin{tabular}{|c|c|c|c|c|c|}
\hline & \multicolumn{2}{|c|}{ Position of the center } & \multicolumn{2}{|c|}{ Main axes } & \multirow{2}{*}{$\begin{array}{c}\text { Major axis P.A. } \\
\mathrm{PA}_{0}-3, \mathrm{PA}_{0}\end{array}$} \\
\hline ShaSS 622 & $\mathrm{x}_{0}-1, \mathrm{x}_{0}+1$ & $\mathrm{y}_{0}$ & $\mathrm{a}_{0}, \mathrm{a}_{0}+2$ & $\mathrm{~b}_{0}, \mathrm{~b}_{0}+1$ & \\
\hline ShaSS 073 & $\mathrm{x}_{0}$ & $\mathrm{y}_{0}$ & $\mathrm{a}_{0}-0.5, \mathrm{a}_{0}+0.8$ & $\mathrm{~b}_{0}-0.5, \mathrm{~b}_{0}+0.5$ & $\mathrm{PA}_{0}-3, \mathrm{PA}_{0}+1$ \\
\hline
\end{tabular}

where

$$
\begin{aligned}
& A=h \cdot \tan (\psi) \cdot \cos (\xi) /|D| \\
& B=A \cdot|D|^{\frac{1}{2}}
\end{aligned}
$$

This is the equation of an ellipse or an hyperbola depending on whether $D$ is greater or smaller than zero. From the above relations, the eccentricity $e$ of the conic is simply given by $e^{2}=1-D(e<1$ for an ellipse and $e>1$ for an hyperbola). Notice that by construction the origin of the coordinates is the intersection of the axis of the ionization cone with the plane of ShaSS 622, while the center of the conic is $x_{o}$ (Eq. A6). The distance between the intersection of the cone axis and the focus of the conics is given by $x_{0}-\operatorname{sgn}(D) \cdot A \cdot e$ for the ellipse and hyperbola and $A_{p a r}-h / 2 \sin (\xi)$, where $A_{\text {par }}=\frac{1}{2} \cdot h \cdot \tan (\psi)^{2} \cdot \sin (\xi)$ is the distance from the focus to the vertex of the parabola. The relations derived for the ionization cone, combined with the preceding ones, allow to determine the other quantities present in Table 4.

To asses the reliability of the parameters defining the three-dimensional structure of the system (Table 4), we repeated the above derivations with different sets of initial parameters for the two disks - position, ellipticity and orientation. We considered acceptable values of the parameters for ShaSS 622 those for which the gas velocity field remains fairly symmetric with respect to the center of the galaxy. For ShaSS 073 these parameters are well constrained by the unambiguous definition of the center and the regular (elliptical) shape of the external isophotes (in the unperturbed region). In any case, we kept the widest possible ranges for all of the parameters in order to stress their influence on the results. The adopted ranges of the initial parameters are listed in Table A1, while Fig. A1 shows the ellipses generated by the parameters within those ranges.

The derived ranges of the parameters defining the spatial structure of the system are given in square brackets in Table 4.

We note that changing the initial values of the ellipses leads to a 7-dimensional parameter space, which is impossible to keep under control. Furthermore, many parameters are correlated ${ }^{12}$. The limits in Table 4 are instead given as they were independent. A more rigorous treatment is however out of our scope, which is to verify that the values provided by our model are reasonable and robust against reasonable changes of the assumed initial parameters.

The range of cone semi-apertures was derived as follows. For each of the above models, we produced the analogous of Fig. 20, and changed the semi-aperture and the intersection of the cone axis with ShaSS 622 (cyan cross in Fig. 20) to achieve the best match of the theoretical conics with the curves of constant ratios [O III] $/ \mathrm{H} \beta$ (red isophotes in Fig. 20). The vast majority of models return values close to $12^{\circ}$, no model gives lower values than this, while the value of $15^{\circ}$ is produced in just one model. In this case the intersection of the cone axis with the disk of ShaSS 622 (cyan cross in Fig. 20) moves to $\mathrm{y}_{\mathrm{c}}<20$, well outside of the disk.

This analysis leads us to conclude that the model represented by Figs. 21 and 22 is a realistic description of the spatial structure of the ShaSS 073-622 system.

\footnotetext{
12 As an example, the angle $\zeta$ between ShaSS 073 and the cone axis and the angle $\gamma$ between the disks of the two galaxies are anti-correlated, so that there is no system with $\zeta=66^{\circ}$ and $\gamma=22^{\circ}$,
} 


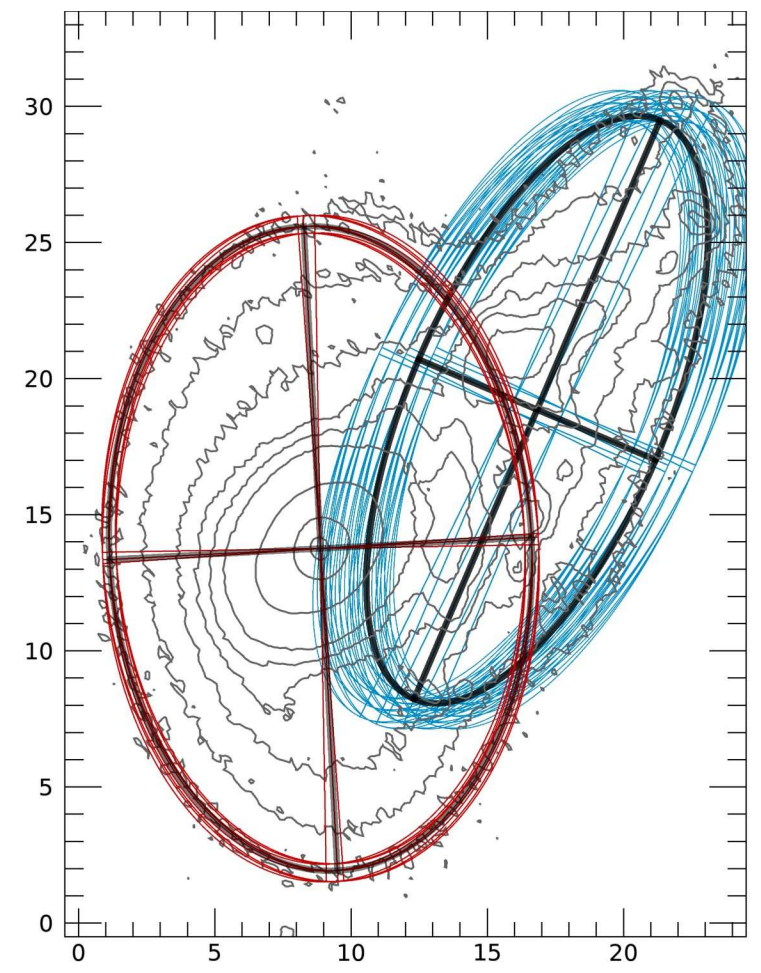

Figure A1. The ellipses (with relative main axes) representing the position, ellipticity, and orientation of the disks of the two galaxies are plotted in black over the $r$-band isophotes of the ShaSS 073-622 system, in the same way as in Fig 19. The ellipses corresponding to the variants of these parameters adopted for the present analysis are shown in blue for ShaSS 622 and red for ShaSS 073. These values are used to estimate the uncertainties on the three-dimensional structure of the system, as explained in the text.

\section{REFERENCES}

Bae, H.-J., \& Woo, J.-H. 2016, ApJ, 828, 97

Baldwin, J. A., Phillips, M. M., \& Terlevich, R. 1981, PASP, 93, 5

Bell, E. F., \& de Jong, R. S. 2001, ApJ, 550, 212

Blanc, G. A., Kewley, L., Vogt, F. P. A., \& Dopita, M. A. 2015, ApJ, 798, 99

Buta, R. J. 2013, Galaxy Morphology, ed. T. D. Oswalt \& W. C. Keel, 1

Cardamone, C., Schawinski, K., Sarzi, M., et al. 2009, MNRAS, 399,1191

Childress, M. J., Vogt, F. P. A., Nielsen, J., \& Sharp, R. G. 2014, Ap\&SS, 349, 617

Condon, J. J., Cotton, W. D., Greisen, E. W., et al. 1998, AJ, 115,1693

Conselice, C. J. 2014, ARA\&A, 52, 291

Crenshaw, D. M., Kraemer, S. B., Hutchings, J. B., et al. 2000, AJ, 120, 1731

Dale, D. A., \& Helou, G. 2002, ApJ, 576, 159

Dale, D. A., Helou, G., Magdis, G. E., et al. 2014, ApJ, 784, 83

Davies, L. J. M., Robotham, A. S. G., Driver, S. P., et al. 2015a, MNRAS, 452, 616

Davies, R. L., Schirmer, M., \& Turner, J. E. H. 2015b, MNRAS, 449, 1731

Davies, R. L., Dopita, M. A., Kewley, L., et al. 2016, ApJ, 824, 50

Diehl, S., \& Statler, T. S. 2006, MNRAS, 368, 497

Donley, J. L., Koekemoer, A. M., Brusa, M., et al. 2012, ApJ, 748,142

Dopita, M., Hart, J., McGregor, P., et al. 2007, ApSS, 310, 255

Dopita, M., Rhee, J., Farage, C., et al. 2010, ApSS, 327, 245
Dopita, M. A., Sutherland, R. S., Nicholls, D. C., Kewley, L. J., \& Vogt, F. P. A. 2013, ApJS, 208, 10

Dopita, M. A., Scharwächter, J., Shastri, P., et al. 2014, A\&A, 566, A41

Fabian, A. C. 2012, ARA\&A, 50, 455

Fischer, T. C., Crenshaw, D. M., Kraemer, S. B., \& Schmitt, H. R. 2013, ApJS, 209, 1

Fischera, J., \& Dopita, M. 2005, ApJ, 619, 340

Fu, H., \& Stockton, A. 2009, ApJ, 690, 953

Fukugita, M., Shimasaku, K., \& Ichikawa, T. 1995, PASP, 107, 945

Grado, A., Capaccioli, M., Limatola, L., \& Getman, F. 2012, Mem. SAIt, 19, 362

Gunn, J. E., \& Gott, J. R. I. 1972, ApJ, 176, 1

Haines, C. P., Busarello, G., Merluzzi, P., et al. 2011, MNRAS, 412,145

Hainline, K. N., Hickox, R. C., Greene, J. E., et al. 2014, ApJ, 787,65

Harrison, C. M., Alexander, D. M., Mullaney, J. R., \& Swinbank, A. M. 2014, MNRAS, 441, 3306

Ho, I.-T., Medling, A. M., Groves, B., et al. 2016, ApSS, 361, 280

Hopkins, P. F., Somerville, R. S., Cox, T. J., et al. 2009, MNRAS, 397, 802

Ichikawa, K., \& Tazaki, R. 2017, ApJ, 844, 21

Jarrett, T. H., Cohen, M., Masci, F., et al. 2011, ApJ, 735, 112

Józsa, G. I. G., Garrett, M. A., Oosterloo, T. A., et al. 2009, A\&A, 500, L33

Karouzos, M., Woo, J.-H., \& Bae, H.-J. 2016, ApJ, 819, 148

Kauffmann, G., Heckman, T. M., Tremonti, C., et al. 2003, MNRAS, 346, 1055 
Keel, W. C., Chojnowski, S. D., Bennert, V. N., et al. 2012a, MNRAS, 420, 878

Keel, W. C., Lintott, C. J., Schawinski, K., et al. 2012b, AJ, 144, 66

Keel, W. C., Maksym, W. P., Bennert, V. N., et al. 2015, AJ, 149, 155

Keel, W. C., Lintott, C. J., Maksym, W. P., et al. 2017, ApJ, 835,256

Kewley, L. J., Groves, B., Kauffmann, G., \& Heckman, T. 2006, MNRAS, 372, 961

Kewley, L. J., Heisler, C. A., Dopita, M. A., \& Lumsden, S. 2001, ApJS, 132, 37

King, A., \& Pounds, K. 2015, ARA\&A, 53, 115

Kozłowski, S., Kochanek, C. S., Ashby, M. L. N., et al. 2016, ApJ, 817, 119

Kroupa, P. 2001, MNRAS, 322, 231

Lacy, M., Ridgway, S. E., Sajina, A., et al. 2015, ApJ, 802, 102

Leitherer, C., Schaerer, D., Goldader, J. D., et al. 1999, ApJS, 123,3

Lintott, C. J., Schawinski, K., Keel, W., et al. 2009, MNRAS, 399, 129

Lutz, D., Maiolino, R., Spoon, H. W. W., \& Moorwood, A. F. M. 2004, A\&A, 418, 465

Mateos, S., Carrera, F. J., Alonso-Herrero, A., et al. 2015, MNRAS, 449, 1422

McElroy, R., Croom, S. M., Pracy, M., et al. 2015, MNRAS, 446,2186

Mercurio, A., Merluzzi, P., Busarello, G., et al. 2015, MNRAS, 453, 3685

Merluzzi, P., Busarello, G., Dopita, M. A., et al. 2016, MNRAS, 460,3345

-. 2013, MNRAS, 429, 174

Merluzzi, P., Busarello, G., Haines, C. P., et al. 2015, MNRAS, 446, 803

Moshir, M., Kopan, G., Conrow, T., et al. 1990, in BAAS, Vol. 22, Bulletin of the American Astronomical Society, 1325

Netzer, H. 2015, ARA\&A, 53, 365

Neugebauer, G., Habing, H. J., van Duinen, R., et al. 1984, ApJL, 278, L1
Nicholls, D. C., Sutherland, R. S., Dopita, M. A., Kewley, L. J., \& Groves, B. A. 2017, MNRAS, 466, 4403

Osterbrock, D. E., \& Ferland, G. J. 2006, Astrophysics of gaseous nebulae and active galactic nuclei

Polletta, M., Tajer, M., Maraschi, L., et al. 2007, ApJ, 663, 81

Rampadarath, H., Garrett, M. A., Józsa, G. I. G., et al. 2010, A\&A, 517, L8

Richards, G. T., Lacy, M., Storrie-Lombardi, L. J., et al. 2006, ApJS, 166, 470

Rieke, G. H., Alonso-Herrero, A., Weiner, B. J., et al. 2009, ApJ, 692, 556

Rubin, V. C., Waterman, A. H., \& Kenney, J. D. P. 1999, AJ, 118,236

Sartori, L. F., Schawinski, K., Koss, M., et al. 2016, MNRAS, 457,3629

Schirmer, M., Diaz, R., Holhjem, K., Levenson, N. A., \& Winge, C. 2013, ApJ, 763, 60

Shi, Y., Helou, G., Armus, L., Stierwalt, S., \& Dale, D. 2013, ApJ, 764, 28

Smith, B. J., Zaragoza-Cardiel, J., Struck, C., Olmsted, S., \& Jones, K. 2016, AJ, 151, 63

Stern, D., Eisenhardt, P., Gorjian, V., et al. 2005, ApJ, 631, 163

Stern, D., Assef, R. J., Benford, D. J., et al. 2012, ApJ, 753, 30

Stockton, A., Fu, H., \& Canalizo, G. 2006, NewAR, 50, 694

Taylor, E. N., Hopkins, A. M., Baldry, I. K., et al. 2011, MNRAS, 418, 1587

Thomas, A. D., Groves, B. A., Sutherland, R. S., et al. 2016, ApJ, 833, 266

Toomre, A., \& Toomre, J. 1972, ApJ, 178, 623

Vazdekis, A., Sánchez-Blázquez, P., Falcón-Barroso, J., et al. 2010, MNRAS, 404, 1639

Veilleux, S., \& Osterbrock, D. E. 1987, ApJS, 63, 295

Véron-Cetty, M.-P., \& Véron, P. 2001, A\&A, 374, 92

Wang, J., Hammer, F., Athanassoula, E., et al. 2012, A\&A, 538, A121

Woo, J.-H., Bae, H.-J., Son, D., \& Karouzos, M. 2016, ApJ, 817, 108

Wright, E. L., Eisenhardt, P. R. M., Mainzer, A. K., et al. 2010, AJ, 140, 1868

Yun, M. S., Reddy, N. A., \& Condon, J. J. 2001, ApJ, 554, 803 